\title{
SUPPORTING INFORMATION Revisiting vertical models to simulate the lineshape of electronic spectra adopting Cartesian and internal coordinates
}

\author{
Javier Cerezo, ${ }^{1, *}$ Fabrizio Santoro ${ }^{1, *}$ \\ ${ }^{1}$ Istituto di Chimica dei Composti OrganoMetallici, \\ Consiglio Nazionale delle Richerche (ICCOM-CNR), \\ Area della Ricerca, via G. Moruzzi 1, I-56124 Pisa, Italy \\ July 22, 2016
}

${ }^{*}$ Corresponding authors: javier.cerezo@pi.iccom.cnr.it, fabrizio.santoro@pi.iccom.cnr.it 


\section{S1 Normal mode analysis with linear coordinates: $\mathrm{x}$ - and $\mathrm{Q}_{1}$-spaces}

\section{S1.1 Cartesian coordinates (x-space)}

If one works with Cartesian coordinates, before performing the frequency analysis, the coordinate frame must be rotated so as to project out the translational and rotational degrees of freedom. This is usually done in mwc coordinates, $\mathbf{q}$, by imposing the Eckart-Sayvetz conditions. ${ }^{1,2}$ Such a rotation can be expressed as, ${ }^{3}$

$$
\mathrm{q}=\mathrm{Dt}
$$

where $\mathbf{D}$ is an orthogonal transformation and $\mathbf{t}=\left\{\mathbf{t}_{t r}, \mathbf{t}_{r o t}, \mathbf{t}_{v i b}\right\}^{t}$ are the Eckart coordinates, which separate the infinitesimal translations $\left(\mathbf{t}_{t r}\right)$, and rotations $\left(\mathbf{t}_{r o t}\right)$ from the internal coordinates $\left(\mathbf{t}_{v i b}\right)$. The vibrational analysis is then performed using only the internal coordinates $\mathbf{t}_{v i b}$, taking the corresponding $\left(N_{v i b} \times 3 N_{a t}\right)$ block of the $\mathbf{D}$ matrix, $\mathbf{D}_{v i b}$. However, given the rectilinear nature of Cartesian coordinates, the definition of the rotational coordinates $\mathbf{t}_{\text {rot }}$ does not correspond to the true rotations of the system. The resulting pseudo-rotations are only associated to zero frequency at stationary points ${ }^{4}$ while, at non-stationary points, they exhibit non-vanishing frequencies that are a signature of the incomplete separation from the internal coordinates. ${ }^{5}$ As a consequence, it also happens that, at non-stationary points, the $N_{v i b}$ vibrational frequencies (mainly the low ones) obtained by the diagonalization of $\mathbf{H}_{t, v i b}=\mathbf{D}_{v i b}^{t} \mathbf{H}_{x} \mathbf{D}_{v i b}$ can be contaminated by the effect of the rotations.

If the inaccuracies just described are disregarded, the standard vibrational analysis can be performed, considering $\mathbf{D}$ independent of the coordinates, and using the internal $\mathbf{t}_{v i b}$-frame,

$$
\begin{aligned}
2 T & =\dot{\mathbf{t}}_{v i b}^{t} \dot{\mathbf{t}}_{v i b} \\
2 V & =\mathbf{t}_{v i b}^{t} \mathbf{H}_{t, v i b} \mathbf{t}_{v i b}+2 \mathbf{g}_{t, v i b}^{t} \mathbf{t}_{v i b}+2 V_{0}
\end{aligned}
$$

where the vib subscript indicates that only the internal part of the Eckart frame is used and, for the sake of brevity, it will be skipped in the following.

The diagonalization of $\mathbf{H}_{t}$ determines the directions of the normal modes of the final state, $\mathbf{L}_{2}\left(\mathbf{q}=\mathbf{L}_{2} \mathbf{Q}_{2}^{\prime \prime}\right)$. On the other hand, the modes of the initial state, $\mathbf{L}_{1}\left(\mathbf{q}=\mathbf{L}_{1} \mathbf{Q}_{1}\right)$, can be straightforwardly evaluated with standard procedures. Therefore, the Duschinsky matrix can be computed from,

$$
\mathbf{J}=\mathbf{L}_{1}^{t} \mathbf{L}_{2}
$$


Notice that $\mathbf{Q}^{\prime \prime}{ }_{2}$ correspond to the final state normal modes, but shifted to the initial state equilibrium geometry. The actual final state normal modes are then, $\mathrm{Q}_{2}=\mathrm{Q}^{\prime \prime}{ }_{2}-\Delta \mathbf{Q}_{2}$, where the structural displacement $\Delta \mathbf{Q}_{2}$ can be obtained by locating the minimum of the final-state quadratic PES using the diagonal Hessian $\left(\boldsymbol{\Lambda}_{2}\right)$ and gradient in terms of the normal modes of the final state,

$$
\Delta \mathbf{Q}_{2}=-\boldsymbol{\Lambda}_{2}^{-1} \mathbf{g}_{\mathbf{Q}_{2}}=-\boldsymbol{\Lambda}_{2}^{-1} \mathbf{L}_{2}^{t} \mathbf{M}^{1 / 2} \mathbf{g}_{x}
$$

and then rotating to the initial state normal mode space to get the normal mode shift vector,

$$
\mathbf{K}=\mathbf{J} \Delta \mathbf{Q}=-\mathbf{J} \boldsymbol{\Lambda}_{2}^{-1} \mathbf{L}_{2}^{t} \mathbf{M}^{1 / 2} \mathbf{g}_{x}
$$

In summary, unlike in the s-space, in the above procedure the rotations defined in Cartesian coordinates cannot be exactly separated from the internal coordinates. This introduces some issues in the Hessian in the Eckart frame which potentially affect the vibrational analysis. In the s-space, the separation is achieved by construction of the curvilinear coordinates. In principle, a possible cure for the problem met in the $\mathbf{x}$-space would be to consider the Eckart coordinates $\mathbf{t}_{v i b}$ as a curvilinear set, i.e. $\mathbf{D}$ dependent on the coordinates, defined in order to exactly separate the vibrations from the rotations. This, in practice, is what is done within the internal s-frame in the previous section.

\section{S1.2 Normal mode coordinates of initial state ( $Q_{1}$-space)}

The vertical problem can be formulated in a further different way by performing the vibrational analysis of the final state using the normal modes of the initialstate, $\mathbf{Q}_{1}$. In this section we show that this leads to models fully equivalent to those derived either in the $\mathbf{x}$ - or in the s-frames, depending on whether $\mathbf{Q}_{1}$ are considered linear combinations of the former or the latter sets. Since the initial state geometry $\left(\mathbf{x}_{0,1}\right)$ is a minimum of the corresponding PES, $\mathbf{Q}_{1}$ coordinates are obtained with standard normal mode analysis $\left(\mathbf{x}-\mathbf{x}_{0,1}=\mathbf{M}^{-1 / 2} \mathbf{L}_{1} \mathbf{Q}_{1}\right)$. The gradient and Hessian matrices of the final state PES in terms of $\mathbf{Q}_{1}$ can be derived from the Cartesian coordinates counterparts by ${ }^{6}$

$$
\begin{aligned}
& \mathbf{g}_{Q_{1}}=\mathbf{L}_{1}^{t} \mathbf{M}^{-1 / 2} \mathbf{g}_{x} \\
& \mathbf{H}_{Q_{1}}=\mathbf{L}_{1}^{t} \mathbf{M}^{-1 / 2}\left(\mathbf{H}_{x}-\mathbf{g}_{Q_{1}}^{t} \boldsymbol{\zeta}_{x}\right) \mathbf{M}^{-1 / 2} \mathbf{L}_{1}
\end{aligned}
$$

where $\mathbf{L}_{1}\left(3 N_{a t} \times N_{v i b}\right)$ is the normal mode matrix for the initial state, already defined on an internal $\left(N_{v i b}\right)$ space. Notice that we wrote Eq. $7 \mathrm{~b}$ in a quite general fashion, introducing the rank-3 array $\boldsymbol{\zeta}_{x}$ whose elements describe the derivative of 
the transformation matrix $\mathbf{M}^{1 / 2} \mathbf{L}_{1}$ with respect to Cartesian coordinates. Using the expresion for curvilinear internal coordinates in terms of Cartesian ones (Eq. 2 in the paper) and the chain-rule, $\boldsymbol{\zeta}_{x}$ can be connected to the analogous array in internal coordinates, $\boldsymbol{\zeta}_{s}$, as follows,

$$
\boldsymbol{\zeta}_{x}=\mathbf{B}^{t} \boldsymbol{\zeta}_{s} \mathbf{B}+\mathbf{L}_{s, 1}^{-1} \boldsymbol{\beta}
$$

At this point, one can decide to work with normal modes defined as linear combinations of either Cartesian or curvilinear internal coordinates. In each of the two cases, the second derivative term $\zeta$ will be zero for the set of coordinates used to define the normal modes. Therefore, if Cartesian coordinates are used, $\boldsymbol{\zeta}_{x}=0$, and Eq. $7 \mathrm{~b}$ becomes

$$
\mathbf{H}_{Q_{1}^{(l i n)}}=\mathbf{L}_{1}^{t} \mathbf{M}^{-1 / 2} \mathbf{H}_{x} \mathbf{M}^{-1 / 2} \mathbf{L}_{1}
$$

that is exactly the same expression reported by one of us in ref. 6. The derivation can now proceed as done in the $\mathbf{x}$-frame. The results in the two frames are actually fully equivalent as expected from the fact that $\mathbf{Q}_{1}$ and $\mathbf{t}_{v i b}$ sets are related by an orthogonal transformation, the one that diagonalizes the Hessian of the initial state expressed in the $\mathbf{t}_{v i b}$-frame. This equivalence also implies that the two strategies share the same problems arising from the contamination of the Hessian due to the contribution of the rotations. The normal modes for the final state are obtained from the rotation of the initial state modes, $\mathbf{Q}_{2}^{\prime}=\mathbf{J}^{t} \mathbf{Q}_{1}$, that diagonalizes the Hessian matrix in Eq. 9, ${ }^{6}$

$$
\mathbf{J}^{t} \mathbf{H}_{Q_{1}} \mathbf{J}=\Lambda_{2}
$$

where $\Lambda_{2}$ is the diagonal force constant matrix for the final state.

The rotated modes, $\mathbf{Q}_{2}^{\prime}$, correspond to the final state ones, but shifted to the initial state equilibrium geometry. The actual final state normal modes are then, $\mathbf{Q}_{2}=\mathbf{Q}_{2}^{\prime}-\Delta \mathbf{Q}_{2}$. The rotation matrix, $\mathbf{J}$, can be identified as the Duschinsky matrix since it relates the normal modes in the initial and final states. The shift vector $(\mathbf{K})$ is computed from the displacement, expressed in the normal modes of the initial state,

$$
\mathbf{K}=\mathbf{J} \mathbf{Q}_{1}=\mathbf{J}\left[-\boldsymbol{\Lambda}_{2}^{-1} \mathbf{J}^{t} \mathbf{g}_{Q_{1}}\right]=-\mathbf{J} \boldsymbol{\Lambda}_{2}^{-1} \mathbf{J}^{t} \mathbf{g}_{Q_{1}}
$$

and this completes the derivation.

At variance with what done above, let now us consider the $\mathbf{Q}_{1}$ coordinates as a linear combination of the curvilinear internal coordinates $\mathbf{s}$, then we get $\boldsymbol{\zeta}_{s}=0$. 
In that case, using Eqs. $7 \mathrm{~b}$ and 8 the final-state Hessian in terms of the initial state modes is given by,

$$
\mathbf{H}_{Q_{1}^{(c u r v)}}=\mathbf{L}_{1}^{t} \mathbf{M}^{-1 / 2}\left(\mathbf{H}_{x}-\mathbf{g}_{s}^{t} \boldsymbol{\beta}\right) \mathbf{M}^{-1 / 2} \mathbf{L}_{1}
$$

where we took into account that $\mathbf{g}_{Q_{1}}^{t} \mathbf{L}_{s, 1}^{-1}=\mathbf{g}_{s}^{t}$. The derivation can be performed exactly along the same lines followed in the section dedicated to the s-frame. In particular, being $\mathbf{Q}_{1}$ curvilinear, one should consider that the kinetic energy expression depends on the coordinates like it happens for the set (see Eq. 9 in the manuscript). On the contrary, considering the simplified form

$$
2 T=\dot{\mathbf{Q}}_{1}^{t} \dot{\mathbf{Q}}_{1}
$$

is equivalent to the adoption of the approximation $\mathbf{G}=\mathbf{G}_{1, e q}$ matrix in the paper. Such a choice is now implicit in the use of the vibrational normal modes of the initial state (remember that $\mathbf{L}_{1, s}^{t} \mathbf{G}_{1, e q}^{-1} \mathbf{L}_{1, s}=\mathbf{1}$, where $\mathbf{1}$ is the identity matrix, ${ }^{7}$ therefore Eq. 13 is equivalent to Eq. 9 in the paper with $\mathbf{G}=\mathbf{G}_{1, e q}$ ).

Before concluding this section, we should mention that the different vertical approaches described above have been already presented in the literature. ${ }^{6,8-10}$ For instance, the formulation in the $\mathbf{Q}_{1}$-space considered as linear combination of Cartesian coordinates was already reported by one of us in $2012 .{ }^{6}$ Gotze et al. adopted the $\mathbf{x}$ - and $\mathbf{s}$-spaces ${ }^{9}$ but they neglected the derivative of the $\mathbf{B}$ transformation in internal coordinates. This choice implies the use of the linearised internal coordinates $\mathbf{S}$, i.e. linear combinations of the $\mathbf{t}_{v i b}$ set adopted in the $\mathbf{x}$-frame and therefore shares the same issue due to the contamination with rotations. The equivalence between linearised internal and Cartesian coordinates can also be inferred by replacing curvilinear by linearised internal coordinates in Eq. 12 , which involves taking $\boldsymbol{\beta}=0$, eventually leading to the same expression obtained in Cartesian coordinates, Eq. 9. More recently, Baiardi et al. ${ }^{10}$ presented a complete review of both adiabatic and vertical methods formulated in internal coordinates. However, the superior performance of internal coordinates to carry out the vibrational analysis at non-stationary points, which is central to the present work, was not analysed in detail. 


\section{S2 Analysis of rotational and vibrational modes in Cartesian coordinates with adiabatic and vertical models}

In Table S1, we report the results of the vibrational analysis for I and VI conducted with either Cartesian or internal coordinates at either the vertical (FC) geometry or the minimum of the ES PES, where the latter are labelled as $\mathrm{A}(\mathrm{C})$ and $\mathrm{A}(\mathrm{I})$ for Cartesian and internal coordinates, respectively. In the case of Cartesian coordinates, the translational and rotational frequencies are obtained by diagonalizing the corresponding $3 \times 3$ blocks of the Hessian matrix in the Eckart frame, while the vibrations are obtained from the remaining $(3 N-6) \times(3 N-6)$ block. They are removed by construction in the curvilinear internal frame. Using Cartesian coordinates, it is observed that, while the translational modes always have frequencies equal to zero, the rotational modes exhibit non-zero frequencies, which are larger at non-stationary points. The non-zero frequencies at the minimum can be attributed to the approximate convergence towards the minimum in the optimization, which lead to a non-vanishing gradient, and numerical errors related with the numerical integration of the DFT functionals which are discussed in ref. 11. We checked indeed for anthracene that these frequencies decrease by using better integration grids. Regarding the gradient, it was set to zero for the calculations intended at the minimum (adiabatic models). On the contrary, the larger values observed at non-stationary points are expected from theory. ${ }^{5,12,13}$ Regarding the low frequencies assigned to vibrational modes, they are significantly different when using Cartesian coordinates at non-stationary points, thus confirming that, in these cases, they cannot be fully separated from the rotational modes. 
Table S1: Lowest frequencies $\left(\mathrm{cm}^{-1}\right)$ computed at the $\mathrm{FC}$ (vertical (V) analysis) and minimum (Min, adiabatic (A) analysis) structures with either Cartesian (C) or internal (I) coordinates.

\begin{tabular}{|c|c|c|c|c|c|c|c|c|}
\hline & \multicolumn{4}{|c|}{$\bar{I}$} & \multicolumn{4}{|c|}{$\overline{\overline{\text { VI }}}$} \\
\hline & \multicolumn{2}{|c|}{$\mathrm{FC}$} & \multicolumn{2}{|c|}{ Min } & \multicolumn{2}{|c|}{$\mathrm{FC}$} & \multicolumn{2}{|c|}{ Min } \\
\hline & $\mathrm{V}(\mathrm{C})$ & $\mathrm{V}(\mathrm{I})$ & $\mathrm{A}(\mathrm{C})$ & $\mathrm{A}(\mathrm{I})$ & $\mathrm{V}(\mathrm{C})$ & $\mathrm{V}(\mathrm{I})$ & $\mathrm{A}(\mathrm{C})$ & $\mathrm{A}(\mathrm{I})$ \\
\hline $\mathrm{t}$ & 0 & - & 0 & - & 0 & - & 0 & - \\
\hline t & 0 & - & 0 & - & 0 & - & 0 & - \\
\hline $\mathrm{t}$ & 0 & - & 0 & - & 0 & - & 0 & - \\
\hline $\mathrm{r}$ & $45 i$ & - & $8 i$ & - & $21 i$ & - & $3 i$ & - \\
\hline $\mathrm{r}$ & $34 i$ & - & 9 & - & $4 i$ & - & $1 i$ & - \\
\hline $\mathrm{r}$ & 61 & - & 11 & - & 13 & - & 7 & - \\
\hline 1 & $75 i$ & 90 & 89 & 89 & $34 i$ & 5 & 5 & 5 \\
\hline 2 & 140 & 112 & 112 & 112 & $28 i$ & 9 & 9 & 9 \\
\hline 3 & 157 & 237 & 232 & 232 & $5 i$ & 14 & 14 & 14 \\
\hline 4 & 228 & 240 & 239 & 239 & 7 & 16 & 14 & 14 \\
\hline
\end{tabular}




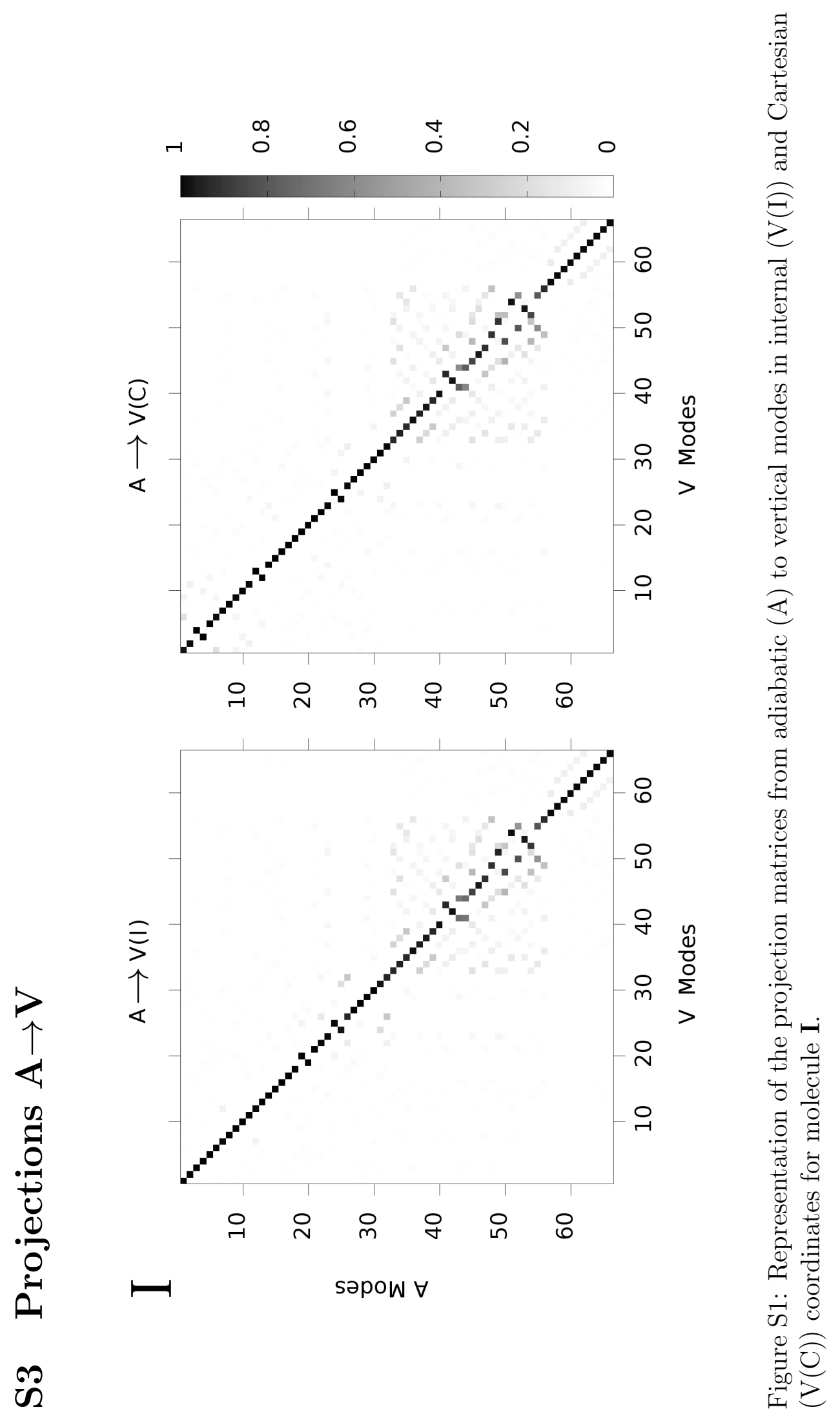




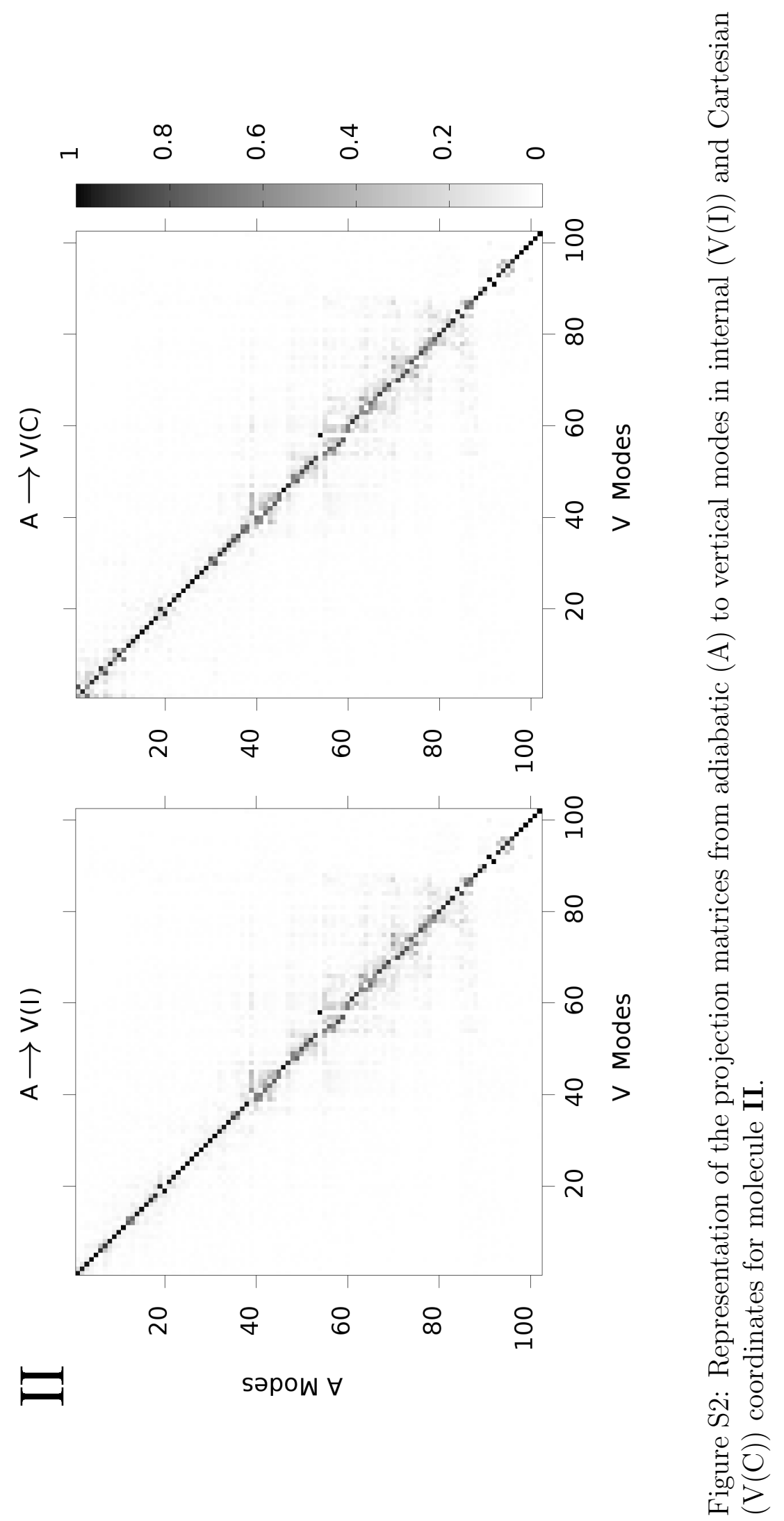




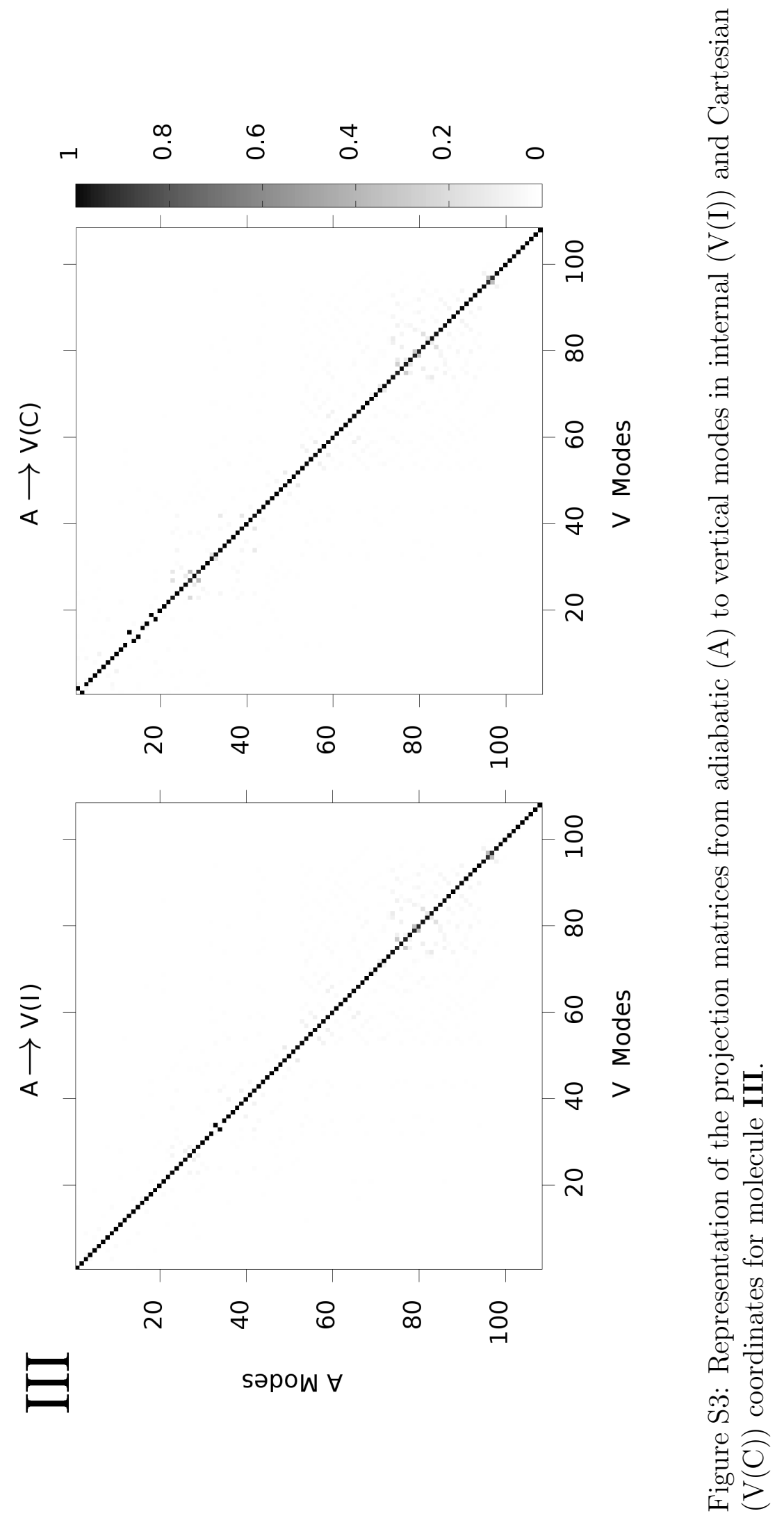




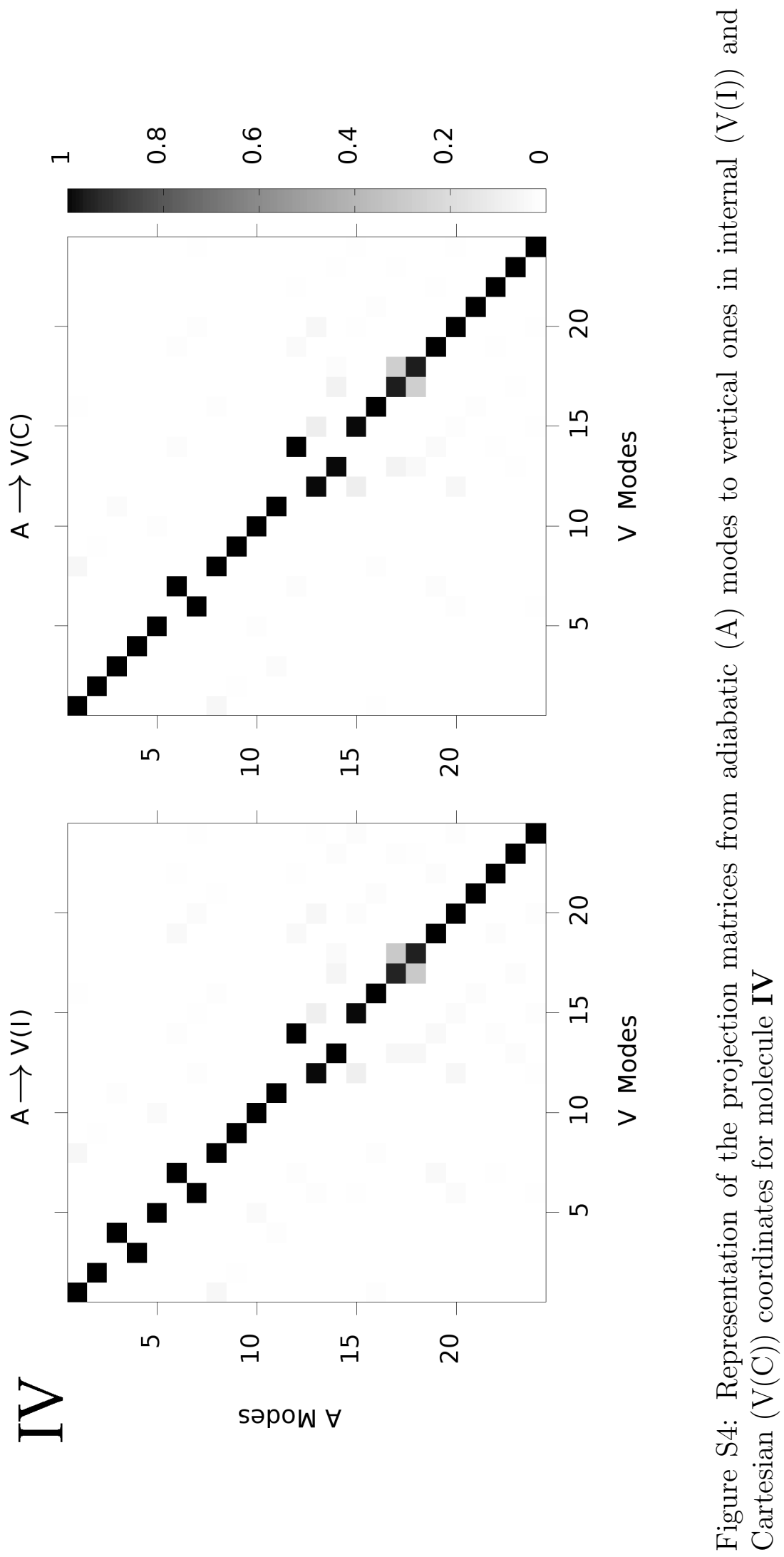




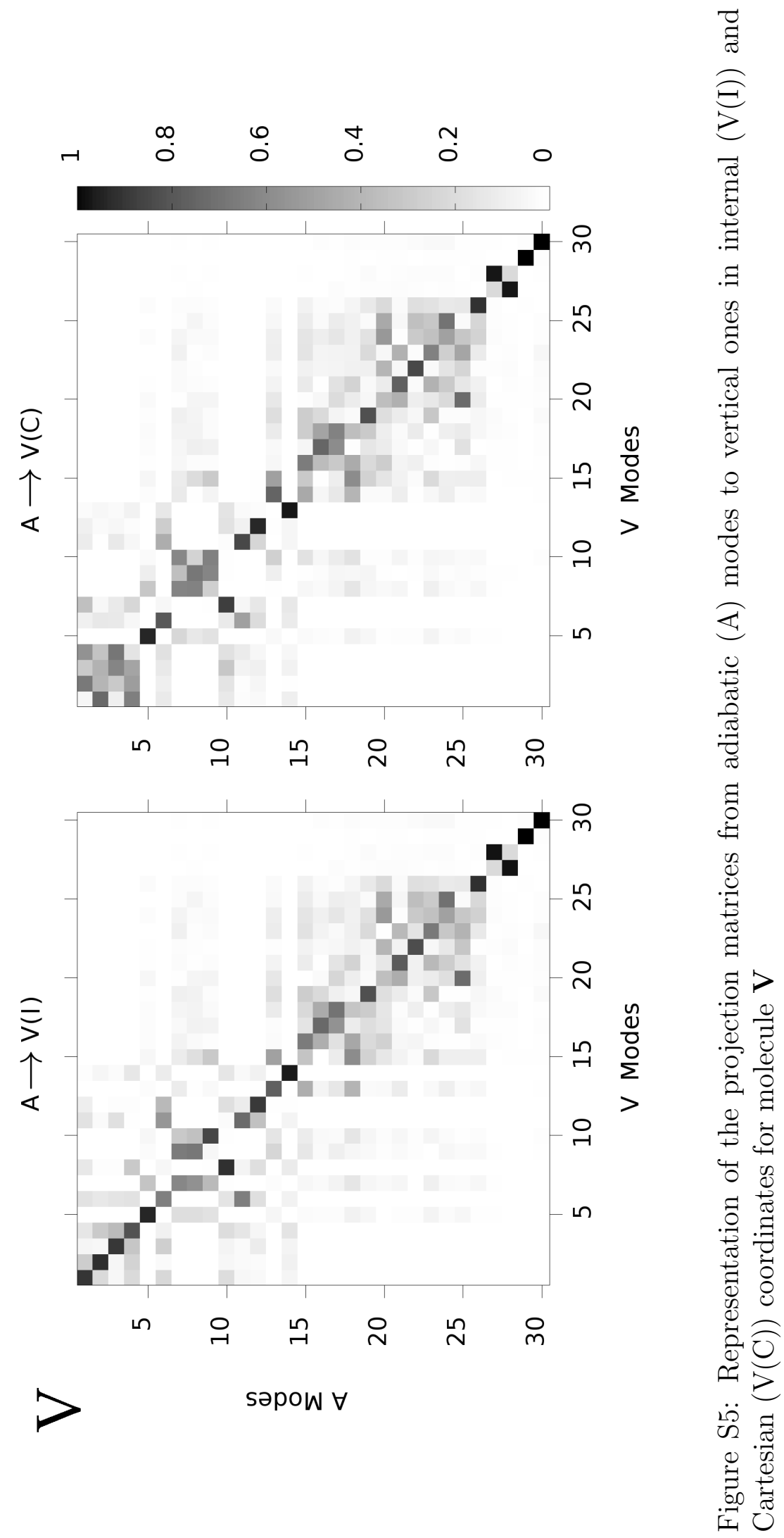




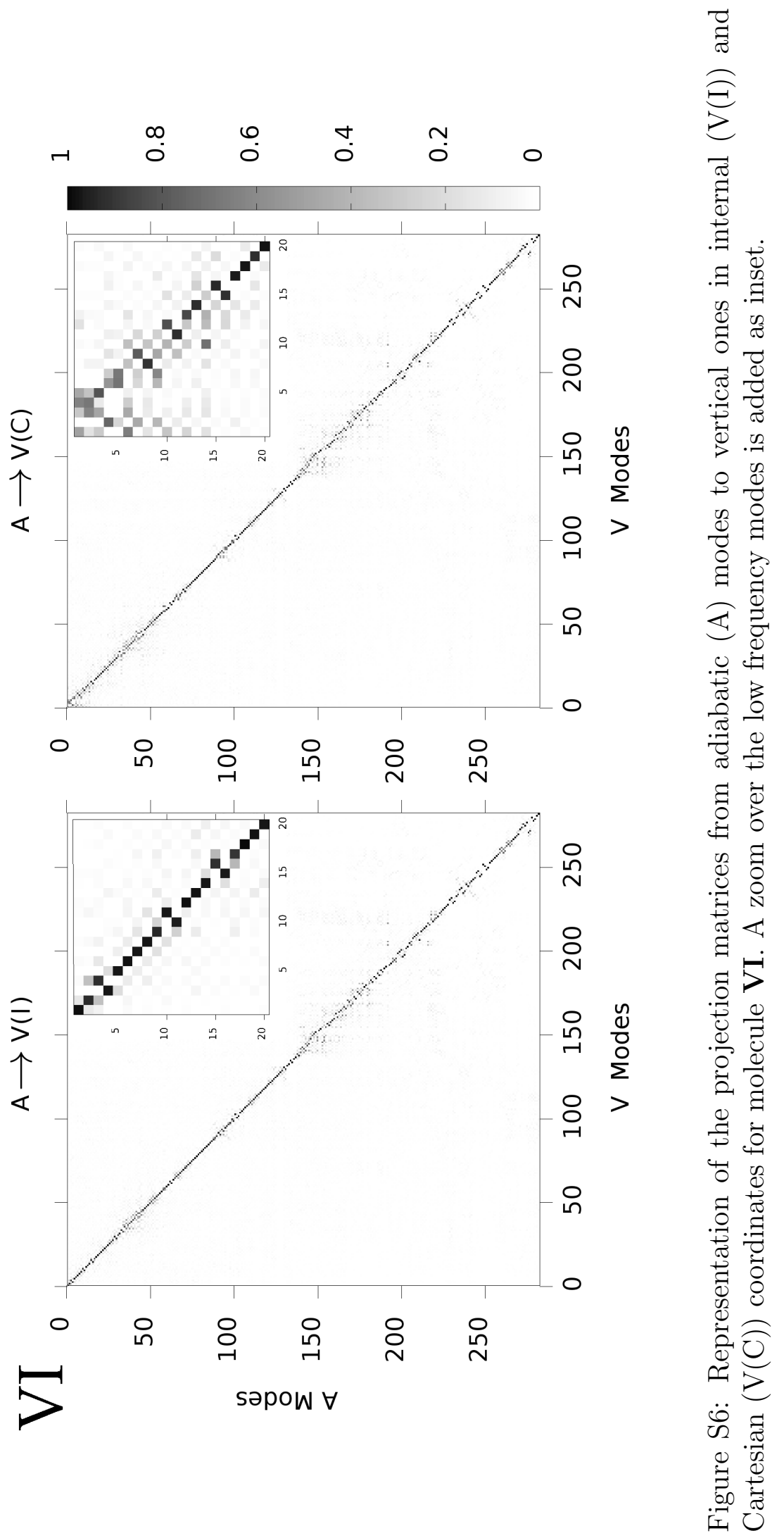




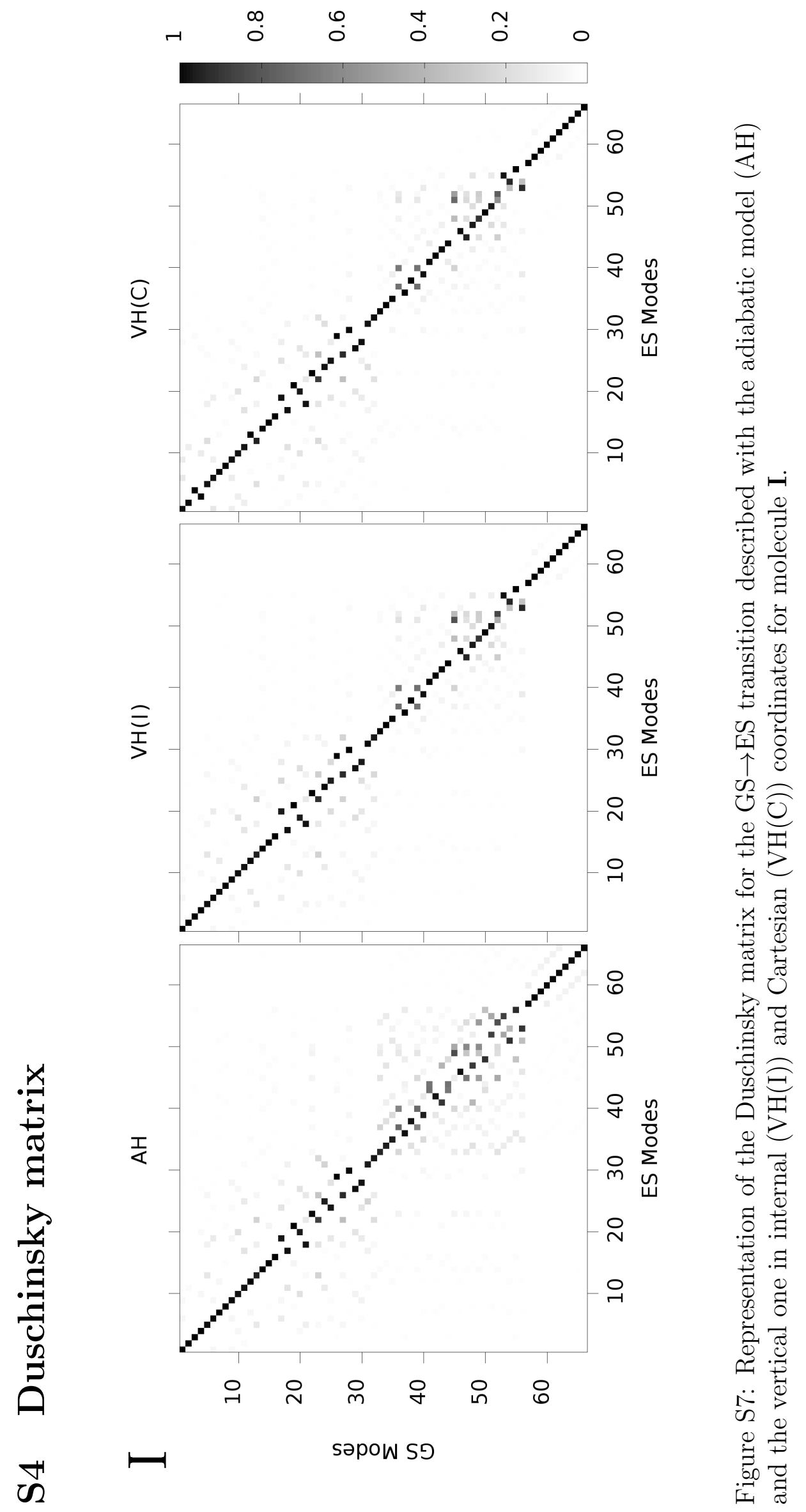




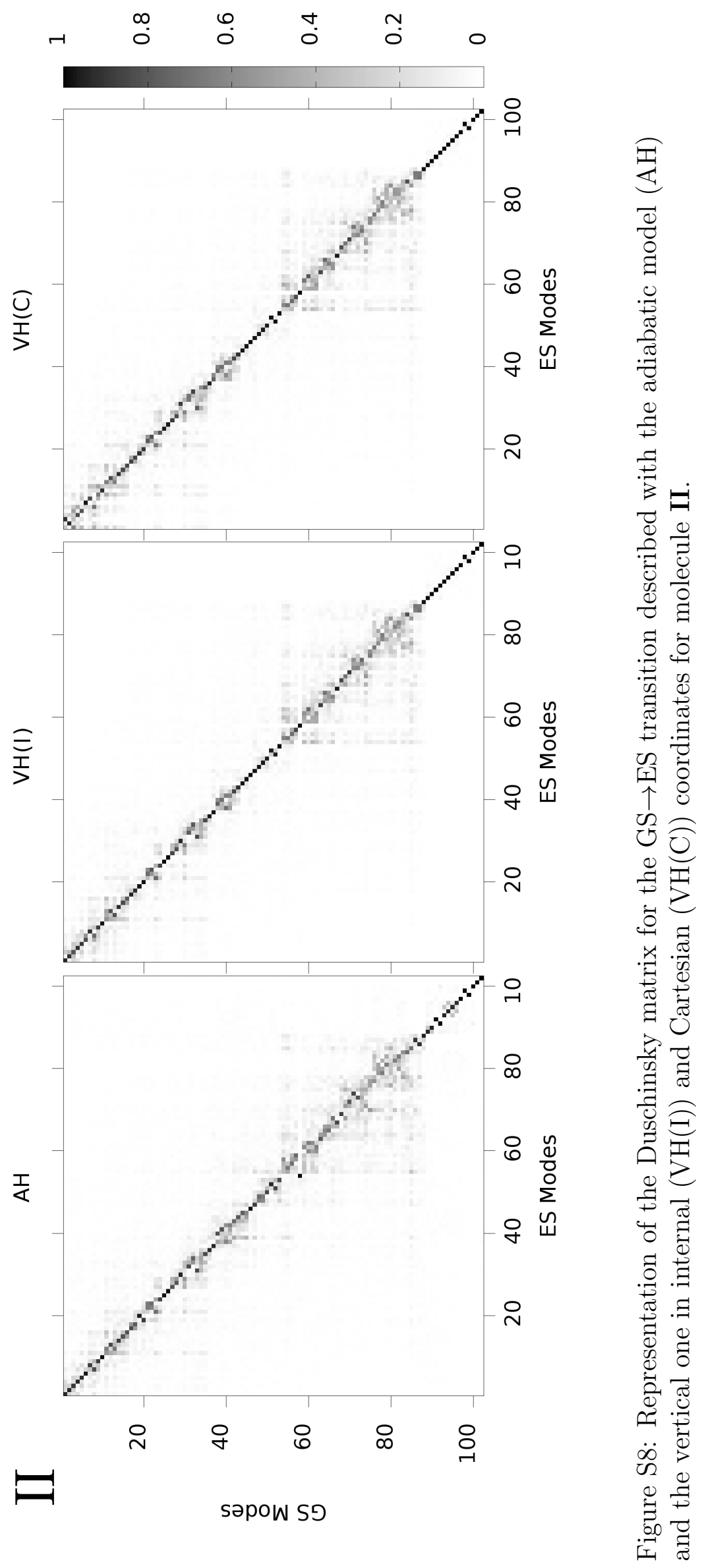




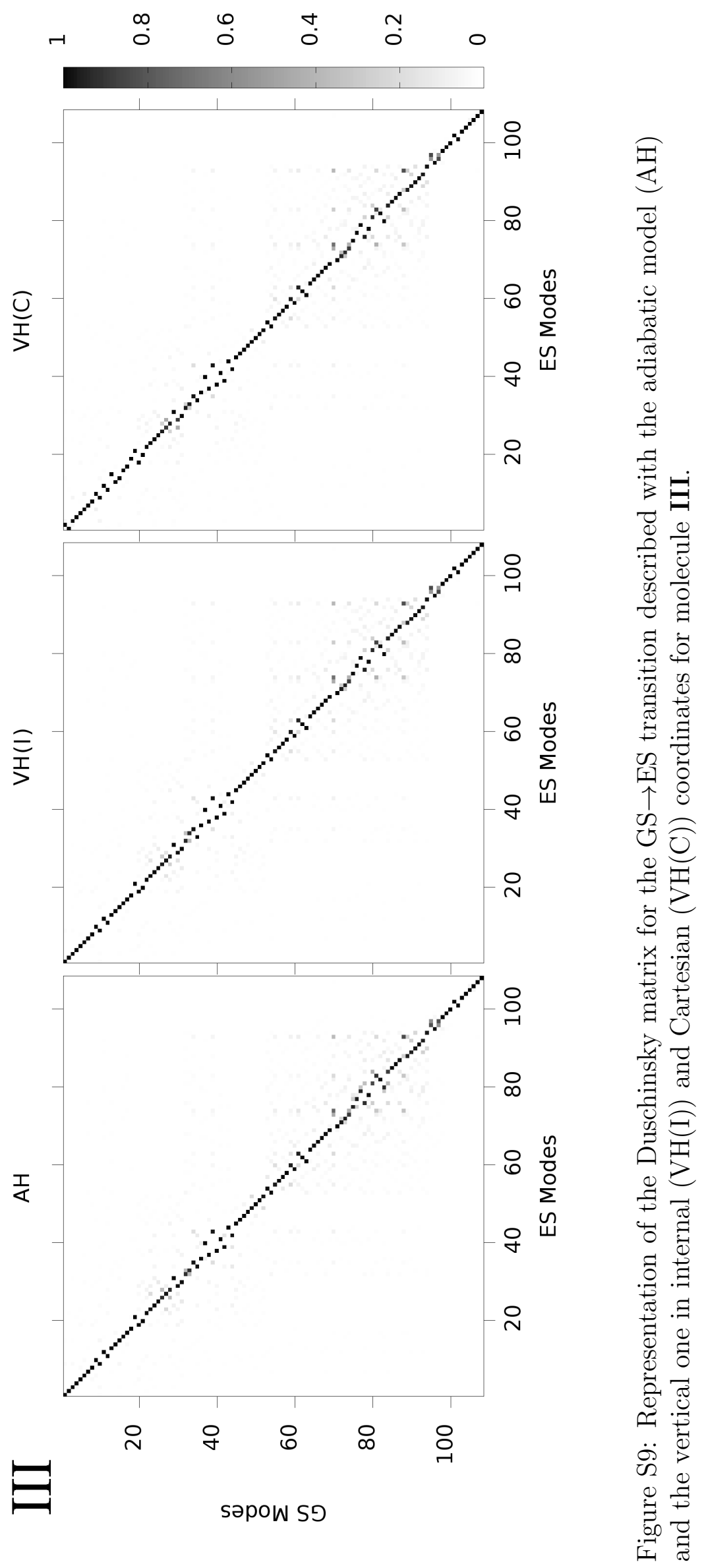




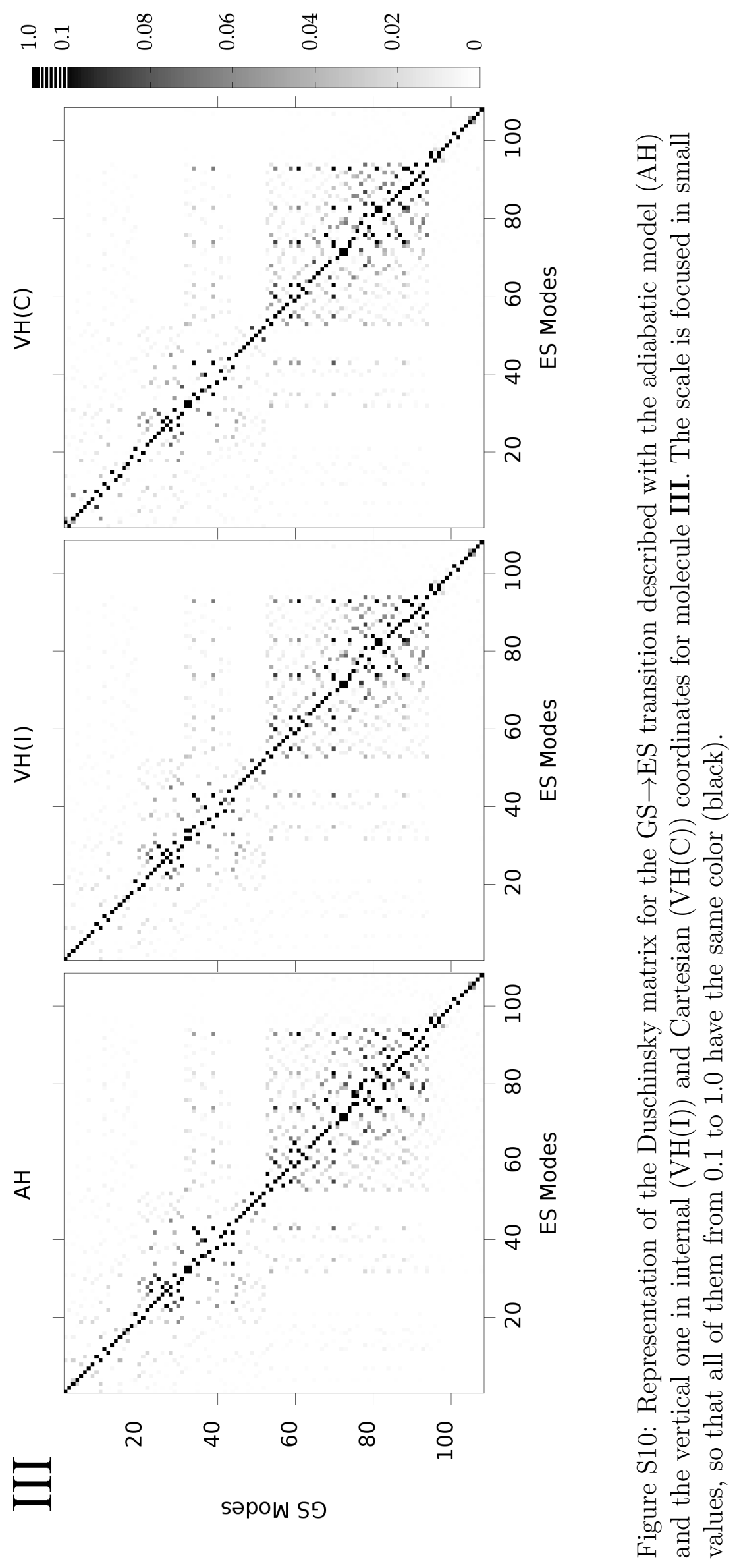



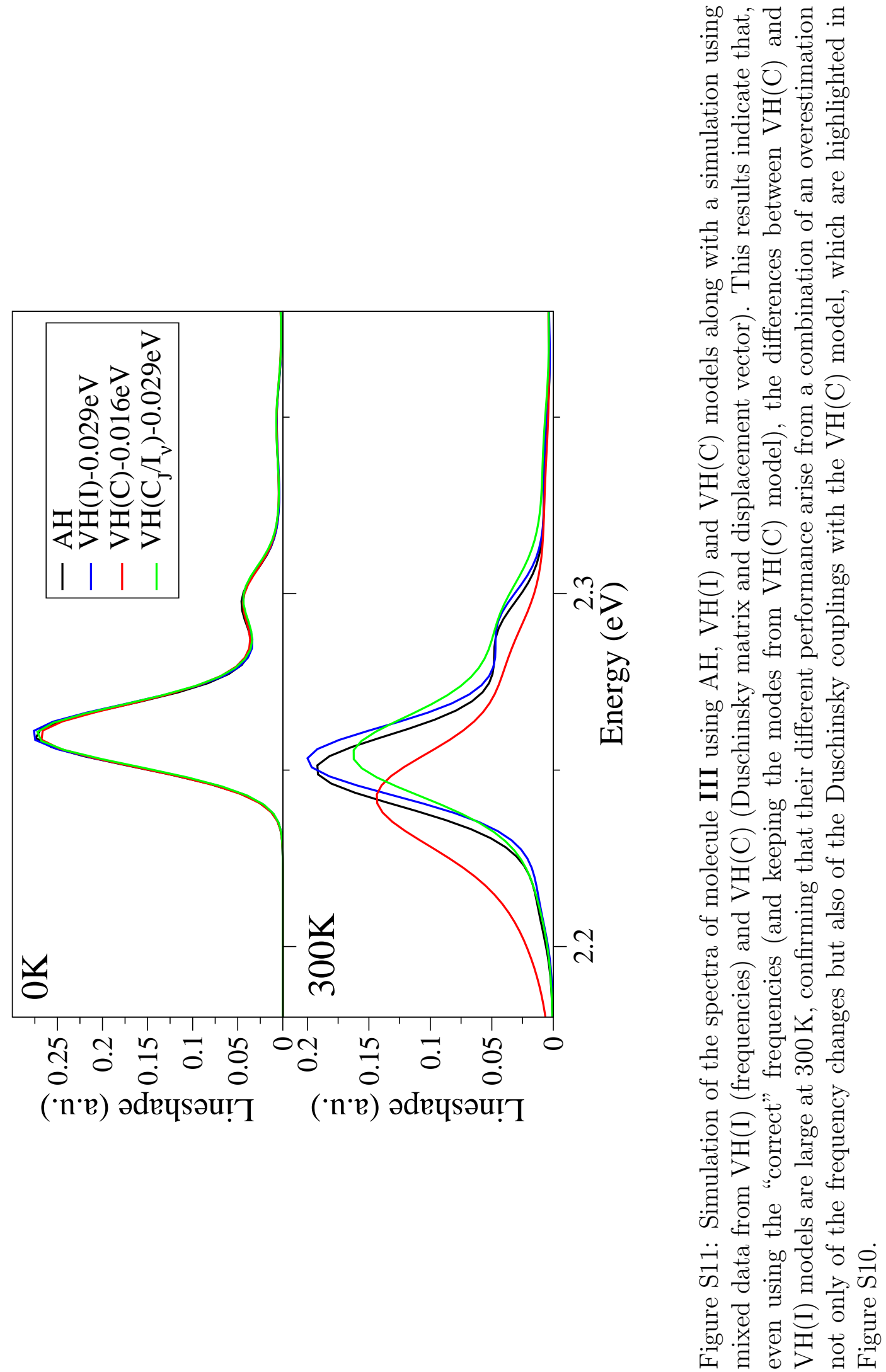


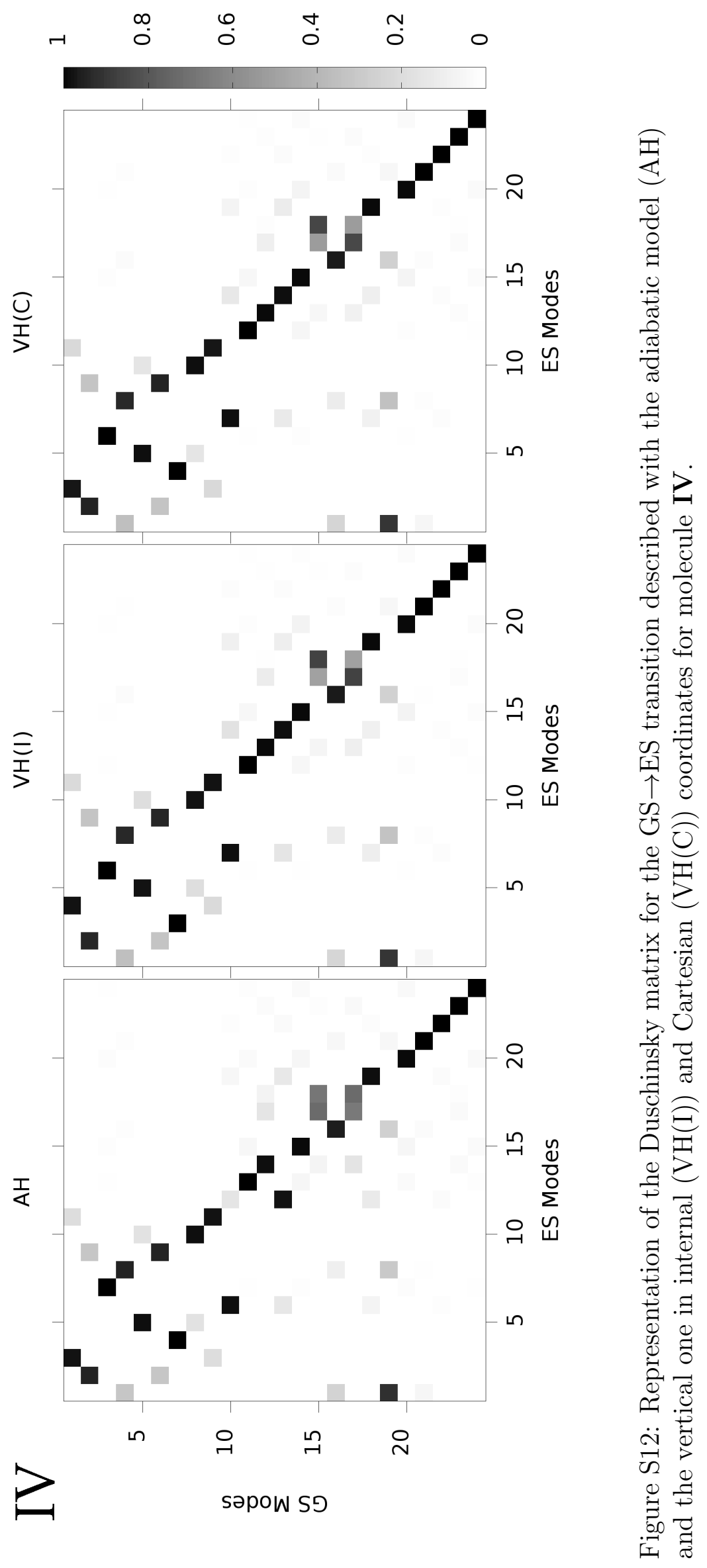




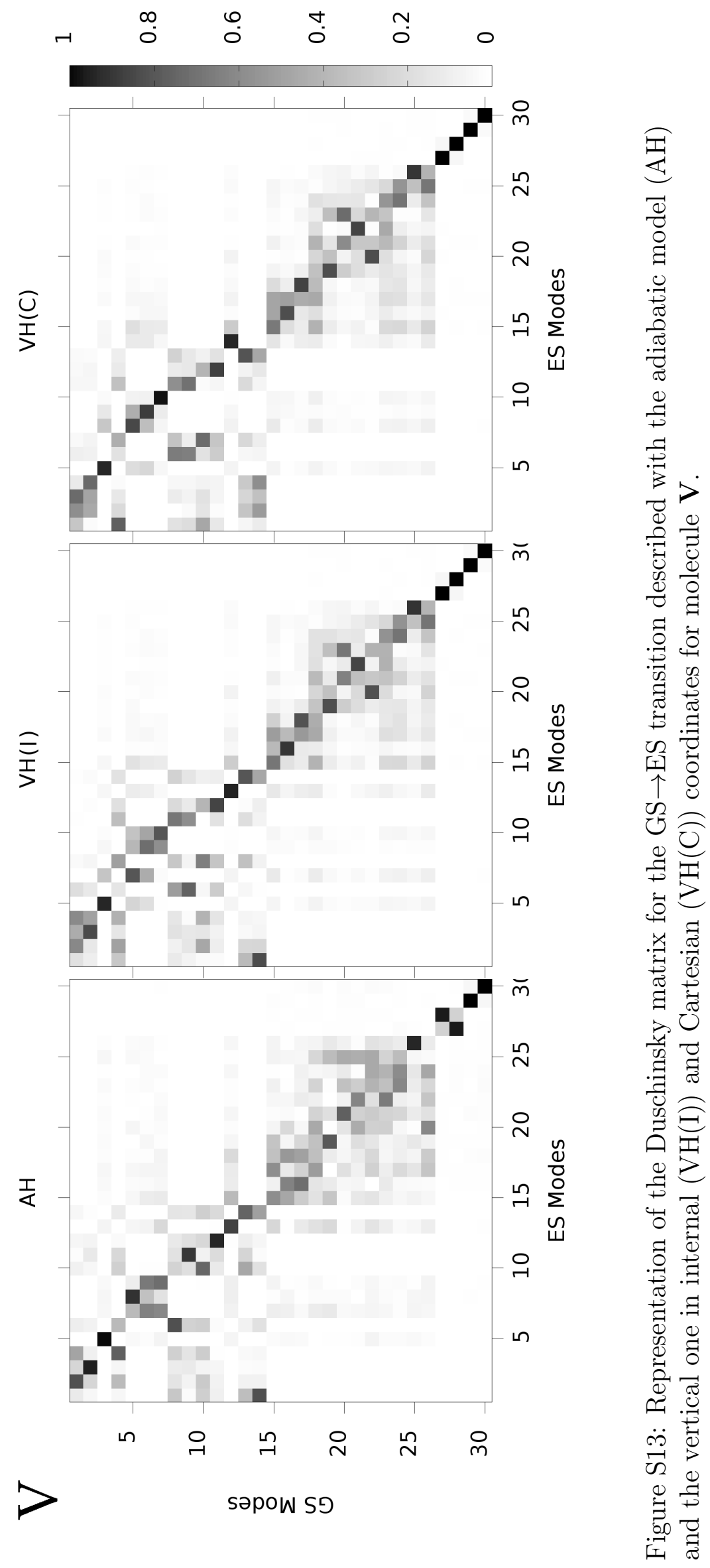



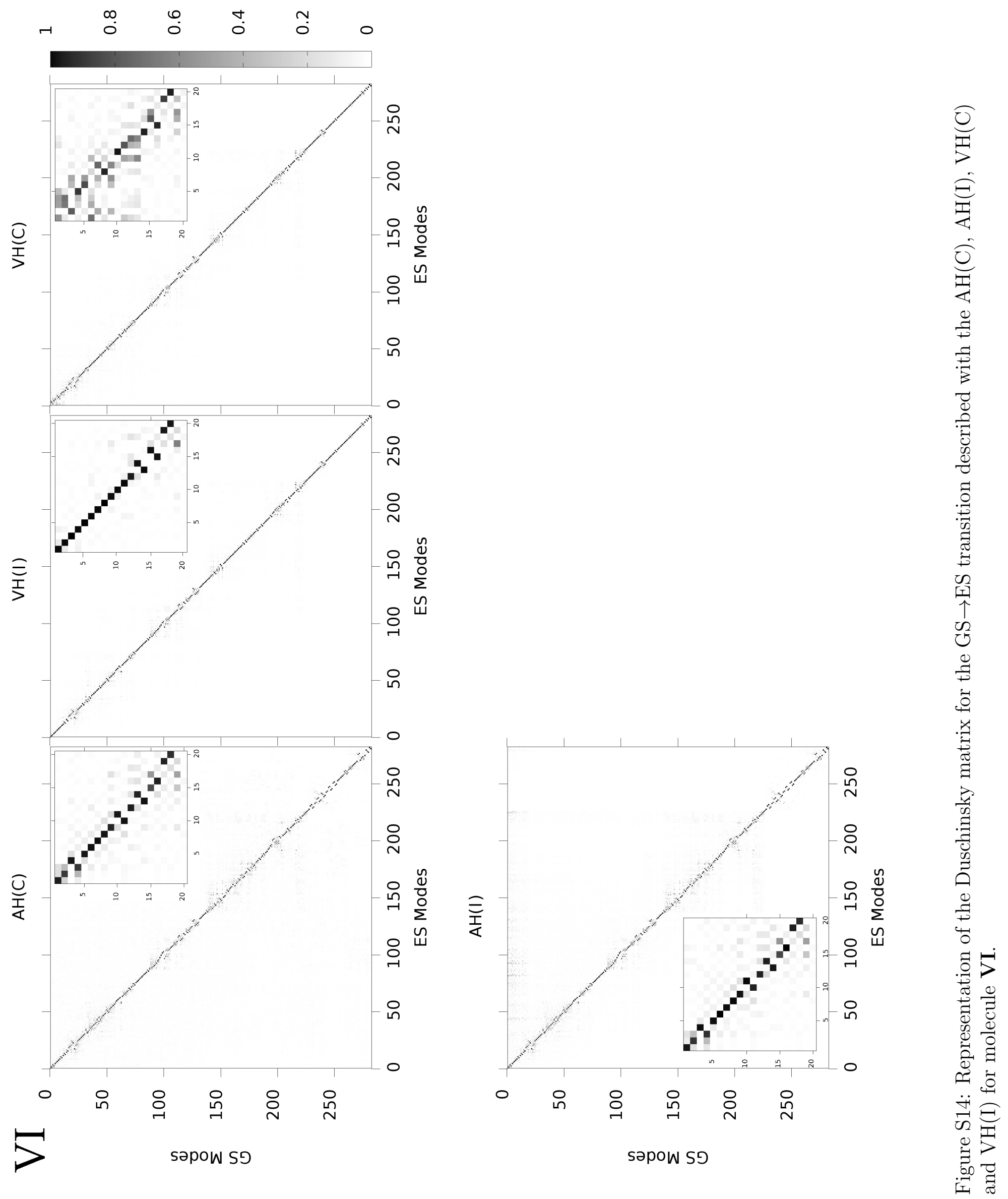


\section{S5 Symmetry of the Hessian in internal coordi- nates}

Considering the Hessian defined in the $\mathbf{Q}_{1}$ space (Eq. (37) in the paper), the

invariance to symmetry operations can be expressed as, $\mathbf{O}_{\hat{R}}^{\left(\mathbf{Q}_{1}\right)} \mathbf{H}_{Q_{1}} \mathbf{O}_{\hat{R}}^{\left(\mathbf{Q}_{1}\right)}=\mathbf{H}_{Q_{1}}$, where $\mathbf{O}_{\hat{R}}^{\left(\mathbf{Q}_{1}\right)}$ is the representation of the symmetry operation on the basis of the normal modes. Applying this transformation to Eq. (37) in the text,

$$
\mathbf{O}_{\hat{R}}^{\left(\mathbf{Q}_{1}\right)} \mathbf{H}_{Q_{1}} \mathbf{O}_{\hat{R}}^{\left(\mathbf{Q}_{1}\right)}=\mathbf{L}_{1}^{t} \mathbf{M}^{-1 / 2} \mathbf{O}_{\hat{R}}^{(\mathbf{x})}\left(\mathbf{H}_{x}-\mathbf{g}_{s}^{t} \boldsymbol{\beta}\right) \mathbf{O}_{\hat{R}}^{(\mathbf{x})} \mathbf{M}^{-1 / 2} \mathbf{L}_{1}
$$

where $\mathbf{O}_{\hat{R}}^{(\mathbf{x})}$ is the representations of the symmetry operation in Cartesian basis, and we used that $\left[\mathbf{M}^{-1 / 2} \mathbf{L}_{1}\right] \mathbf{O}_{\hat{R}}^{\left(\mathbf{Q}_{1}\right)}=\mathbf{O}_{\hat{R}}^{(\mathbf{x})}\left[\mathbf{M}^{-1 / 2} \mathbf{L}_{1}\right]$. The latter property arise from the similarity transformation between the representation in Cartesian and normal modes coordinates. Therefore, in order to comply with the molecular symmetry, $\left(\mathbf{H}_{x}-\mathbf{g}_{s}^{t} \boldsymbol{\beta}\right)$ must be invariant to that rotation,

$$
\mathbf{O}_{\hat{R}}^{(\mathbf{x})}\left(\mathbf{H}_{x}-\mathbf{g}_{s}^{t} \boldsymbol{\beta}\right) \mathbf{O}_{\hat{R}}^{(\mathbf{x})}=\mathbf{O}_{\hat{R}}^{(\mathbf{x})} \mathbf{H}_{x} \mathbf{O}_{\hat{R}}^{(\mathbf{x})}-\mathbf{O}_{\hat{R}}^{(\mathbf{x})}\left(\mathbf{g}_{s}^{t} \boldsymbol{\beta}\right) \mathbf{O}_{\hat{R}}^{(\mathbf{x})}=\mathbf{H}_{x}-\mathbf{O}_{\hat{R}}^{(\mathbf{x})}\left(\mathbf{g}_{s}^{t} \boldsymbol{\beta}\right) \mathbf{O}_{\hat{R}}^{(\mathbf{x})}
$$

where we took into account that the Cartesian Hessian, $\mathbf{H}_{x}$, evaluated at the vertical point is already invariant to the symmetry operations.

Consequently, the symmetry of the Hessian in internal coordinates would keep the same symmetry of the Cartesian Hessian only if the term $\left(\mathbf{g}_{s}^{t} \boldsymbol{\beta}\right)$ remains invariant to the symmetry operations. 


\section{S6 Shift between spectra simulated with adia- batic and vertical models}

The absorption spectra simulated with vertical models seem to be systematically blue-shifted with respect to the adiabatic ones. In order to investigate this effect further, in Table $\mathrm{S} 2$ we include the adiabatic $\left(\mathrm{E}_{a d}\right)$ and $0-0$ transition $\left(\mathrm{E}_{0-0}\right)$ energies computed for all the molecules under study.

The results indicate that, except for molecule $\mathbf{V}$, which is the most flexible one in the ES, the adiabatic energies computed with both adiabatic and vertical models are in very good agreement (deviations below $0.006 \mathrm{eV}$ ). The most similar values are obtained for porphyrin, which turn out to be one of the most rigid molecules in the set.

Table S2: Adiabatic and 0-0 transition energies (in eV) for porphyrin evaluating the final state with $\mathrm{AH}, \mathrm{VH}(\mathrm{I})$ and $\mathrm{VH}(\mathrm{C})$ models.

\begin{tabular}{|c|c|c|c|c|c|c|}
\hline \multirow[b]{2}{*}{ Molecule } & \multicolumn{2}{|c|}{$\overline{\mathrm{AH}}$} & \multicolumn{2}{|c|}{$\mathrm{VH}(\mathrm{I})$} & \multicolumn{2}{|c|}{$\mathrm{VH}(\mathrm{C})$} \\
\hline & $\mathrm{E}_{a d}$ & $\mathrm{E}_{0-0}$ & $\mathrm{E}_{a d}$ & $\mathrm{E}_{0-0}$ & $\mathrm{E}_{a d}$ & $\mathrm{E}_{0-0}$ \\
\hline I & 3.150 & 3.066 & 3.156 & 3.089 & 3.156 & 3.074 \\
\hline II & 3.300 & 3.181 & 3.301 & 3.216 & 3.297 & 3.194 \\
\hline III & 2.319 & 2.260 & 2.320 & 2.289 & 2.319 & 2.275 \\
\hline IV & 3.977 & 3.776 & 3.979 & 3.773 & 3.978 & 3.768 \\
\hline $\mathbf{V}$ & 5.051 & 4.909 & 5.118 & 4.966 & 5.109 & 4.927 \\
\hline VI & 2.117 & 2.057 & 2.123 & 2.078 & 2.114 & 2.050 \\
\hline
\end{tabular}



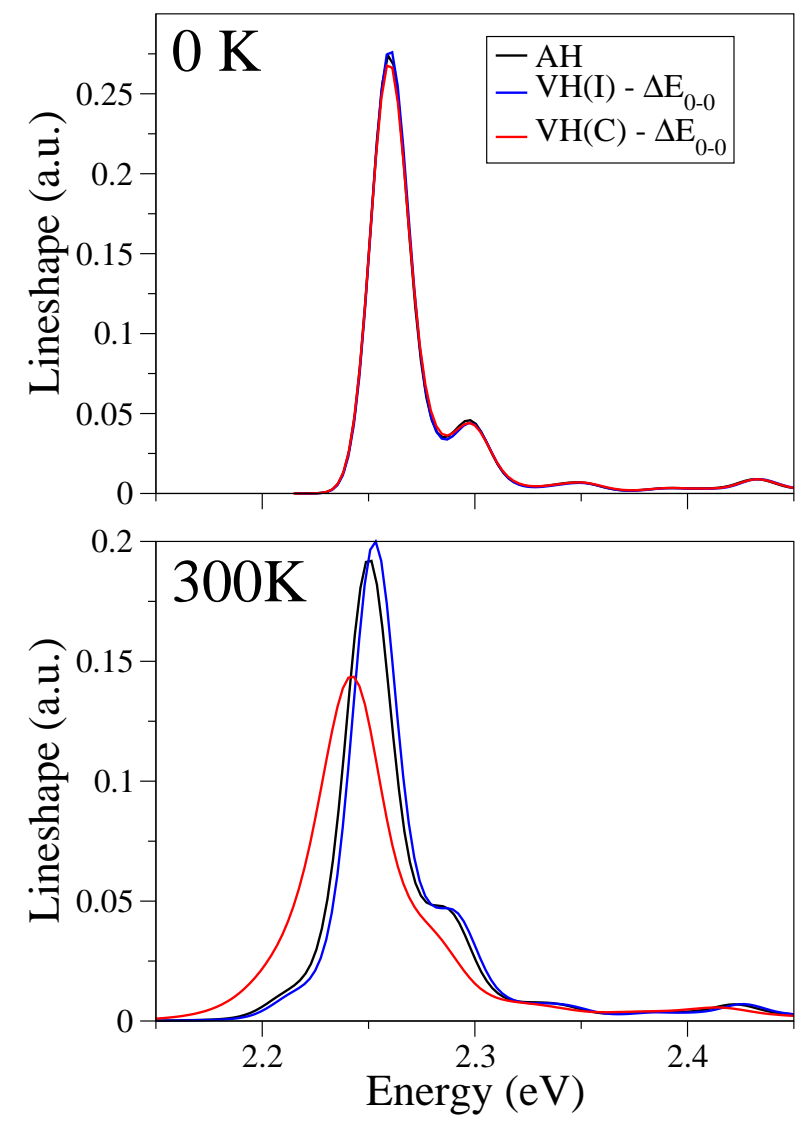

Figure S15: Absorption lineshapes simulated for porphyrin at both 0 and $300 \mathrm{~K}$ using $\mathrm{AH}, \mathrm{VH}(\mathrm{I})$ and $\mathrm{VH}(\mathrm{C})$ models. The vertical spectra are shifted by subtracting $\Delta E_{0-0}=E_{0-0}^{(V H)}-E_{0-0}^{(A H)}$ (energies reported in Table S2).

Finally, we focus on the differences between the position of the spectra of porphyrin, the most rigid molecule. According to the values in Table S2, the differences arise due to the energy of the 0-0 transition. Indeed, if these energies are subtracted the differences with the $\mathrm{AH}$ value for the $\mathrm{VH}(\mathrm{I})$ and $\mathrm{VH}(\mathrm{C})$, both models get over the $\mathrm{AH}$ one at $0 \mathrm{~K}$.

In order to understand the origin of the differences on the 0-0 energies and in Table S3, we report all the frequencies obtained with the $A, V(I)$ and $V(C)$ approaches, including the differences between the vertical and adiabatic results. 
Table S3: Frequencies $\left(\mathrm{cm}^{-1}\right)$ evaluated for porphyrin at the S1 state with adiabatic and vertical models and their differences.

\begin{tabular}{|c|cc|cc|}
\hline \hline $\mathrm{A}$ & $\mathrm{V}(\mathrm{I})$ & $\Delta$ & $\mathrm{V}(\mathrm{C})$ & $\Delta$ \\
\hline 52 & 53 & 1 & 40 & -12 \\
64 & 64 & 0 & 45 & -20 \\
97 & 96 & -1 & 94 & -2 \\
98 & 103 & 4 & 98 & 0 \\
121 & 122 & 1 & 116 & -5 \\
127 & 127 & 0 & 125 & -1 \\
152 & 154 & 2 & 151 & -1 \\
181 & 183 & 1 & 171 & -11 \\
197 & 198 & 1 & 195 & -2 \\
203 & 202 & -1 & 195 & -8 \\
286 & 288 & 1 & 276 & -11 \\
288 & 293 & 5 & 288 & 0 \\
310 & 313 & 3 & 311 & 1 \\
311 & 315 & 4 & 312 & 1 \\
317 & 319 & 1 & 312 & -5 \\
358 & 360 & 2 & 358 & 0 \\
358 & 361 & 3 & 360 & 2 \\
391 & 395 & 4 & 389 & -1 \\
396 & 399 & 3 & 390 & -6 \\
421 & 421 & 0 & 415 & -6 \\
422 & 424 & 2 & 424 & 1 \\
455 & 456 & 1 & 449 & -6 \\
623 & 633 & 10 & 635 & 12 \\
642 & 646 & 5 & 642 & 0 \\
669 & 671 & 2 & 667 & -2 \\
677 & 680 & 3 & 676 & -1 \\
678 & 683 & 5 & 683 & 6 \\
684 & 688 & 4 & 686 & 2 \\
689 & 695 & 5 & 694 & 5 \\
700 & 703 & 3 & 698 & -2 \\
702 & 704 & 3 & 699 & -3 \\
730 & 735 & 5 & 733 & 3 \\
735 & 741 & 6 & 740 & 5 \\
736 & 743 & 6 & 743 & 7 \\
749 & 753 & 4 & 751 & 2 \\
755 & 760 & 6 & 759 & 4 \\
773 & 777 & 4 & 775 & 1 \\
780 & 783 & 3 & 782 & 2 \\
783 & 787 & 4 & 785 & 2 \\
790 & 794 & 4 & 791 & 1 \\
794 & 796 & 2 & 795 & 1 \\
799 & 805 & 5 & 810 & 10 \\
809 & 814 & 5 & 810 & 2 \\
816 & 821 & 5 & 818 & 2 \\
834 & 838 & 4 & 834 & 0 \\
847 & 850 & 4 & 846 & -1 \\
849 & 853 & 4 & 849 & 0 \\
862 & 866 & 4 & 862 & -1 \\
891 & 897 & 6 & 895 & 4 \\
894 & 901 & 7 & 899 & 4 \\
905 & 908 & 3 & 905 & 0 \\
906 & 910 & 4 & 907 & 1 \\
978 & 978 & 0 & 977 & -1 \\
987 & 987 & 1 & 986 & -1 \\
\hline \hline
\end{tabular}

\begin{tabular}{|c|cc|cc|}
\hline \hline $\mathrm{A}$ & $\mathrm{V}(\mathrm{I})$ & $\Delta$ & $\mathrm{V}(\mathrm{C})$ & $\Delta$ \\
\hline 1004 & 1009 & 5 & 1007 & 4 \\
1007 & 1012 & 6 & 1012 & 6 \\
1023 & 1028 & 5 & 1027 & 5 \\
1026 & 1028 & 2 & 1027 & 2 \\
1037 & 1040 & 3 & 1038 & 1 \\
1043 & 1043 & 1 & 1042 & -1 \\
1085 & 1087 & 2 & 1088 & 3 \\
1086 & 1088 & 1 & 1089 & 2 \\
1096 & 1097 & 2 & 1098 & 2 \\
1097 & 1099 & 2 & 1099 & 2 \\
1168 & 1174 & 6 & 1174 & 6 \\
1174 & 1179 & 4 & 1179 & 5 \\
1193 & 1198 & 4 & 1197 & 4 \\
1214 & 1218 & 3 & 1216 & 2 \\
1220 & 1225 & 4 & 1225 & 4 \\
1266 & 1271 & 5 & 1273 & 7 \\
1280 & 1285 & 5 & 1288 & 8 \\
1303 & 1304 & 2 & 1303 & 1 \\
1319 & 1323 & 4 & 1323 & 3 \\
1361 & 1375 & 14 & 1373 & 12 \\
1369 & 1377 & 8 & 1376 & 7 \\
1388 & 1396 & 8 & 1394 & 6 \\
1404 & 1414 & 10 & 1413 & 9 \\
1421 & 1424 & 3 & 1423 & 2 \\
1422 & 1431 & 9 & 1429 & 7 \\
1433 & 1453 & 20 & 1452 & 19 \\
1452 & 1465 & 13 & 1464 & 12 \\
1462 & 1469 & 7 & 1468 & 6 \\
1476 & 1487 & 12 & 1487 & 11 \\
1480 & 1497 & 17 & 1496 & 16 \\
1545 & 1560 & 15 & 1560 & 16 \\
1552 & 1569 & 17 & 1569 & 17 \\
1561 & 1576 & 14 & 1576 & 14 \\
1569 & 1581 & 12 & 1581 & 12 \\
1591 & 1613 & 22 & 1614 & 23 \\
1606 & 1624 & 19 & 1625 & 19 \\
1637 & 1652 & 15 & 1652 & 15 \\
1637 & 1652 & 15 & 1652 & 15 \\
1661 & 1678 & 17 & 1678 & 17 \\
1672 & 1690 & 19 & 1690 & 19 \\
3227 & 3228 & 0 & 3227 & 0 \\
3227 & 3228 & 1 & 3228 & 0 \\
3227 & 3228 & 1 & 3228 & 0 \\
3227 & 3228 & 1 & 3228 & 0 \\
3264 & 3261 & -3 & 3261 & -3 \\
3264 & 3261 & -3 & 3261 & -3 \\
3280 & 3279 & -1 & 3279 & -1 \\
3280 & 3279 & -1 & 3279 & -1 \\
3284 & 3282 & -2 & 3282 & -2 \\
3285 & 3283 & -2 & 3283 & -2 \\
3298 & 3297 & -1 & 3297 & -1 \\
3298 & 3297 & -1 & 3297 & -1 \\
3599 & 3576 & -24 & 3576 & -24 \\
3638 & 3616 & -23 & 3616 & -23 \\
\hline \hline & & & & \\
\hline
\end{tabular}


As shown in this table, the region with the largest differences is that around $1400-1600 \mathrm{~cm}^{-1}$, where the vertical frequencies are $\sim 10-20 \mathrm{~cm}^{-1}$ larger with respect to the adiabatic ones. This explains why the vertical model have a $0-0$ energy that is blue-shifted with respect to the adiabatic value. We should note that this effect is partially counter-balanced by the large difference of the opposite sign that arise for the $\mathrm{N}-\mathrm{H}$ modes around $3600 \mathrm{~cm}^{-1}$. In this case, the difference can be rationalized considering a Morse-like potential to describe the N-H bonding energy. The N-H distance at the vertical point $(1.0154 \AA)$ is slightly larger as compared to the one at the equilibrium geometry in the $\operatorname{ES}(1.0138, \AA)$, and consequently the vertical point is on the attractive part of the potential, which has a curvature (second derivative) that decreases with the distance, i.e., the force constant related the $\mathrm{N}-\mathrm{H}$ bond will be smaller at the vertical point.

Moreover, the difference between the vertical model in internal $(\mathrm{V}(\mathrm{I}))$ and Cartesian $(\mathrm{V}(\mathrm{C})$ ) coordinates can be traced back to the low frequency modes, which are very similar to the adiabatic ones for the $\mathrm{V}(\mathrm{I})$ approach, while being smaller in the case of $\mathrm{V}(\mathrm{C})$ one, which further counter-balance the blue-shift effect and push the 0-0 energy closer to the adiabatic case. Therefore, the good matching between the $E_{00}$ from $\mathrm{V}(\mathrm{C})$ and A models is arising from an error compensation. Figure S16 represents the frequencies with all models and their differences for all molecules studied.

Finally, it can be concluded that the difference between the 0-0 energies computed with vertical and adiabatic model that eventually results in appreciable shifts between the spectra arise from the differences in the frequencies along all the frequency range. We have to consider that since the 0-0 energy depends on the sum over all frequencies, small relative errors in some high frequency modes can actually lead to large fluctuations of the 0-0 energy. Such errors are to be expected since the PES is not harmonic and even for small displacements some modes may display some noticeable differences. It is interesting to note that, as shown in this Figure S16, the frequencies computed with vertical methods are generally larger with respect to those computed with adiabatic ones. 

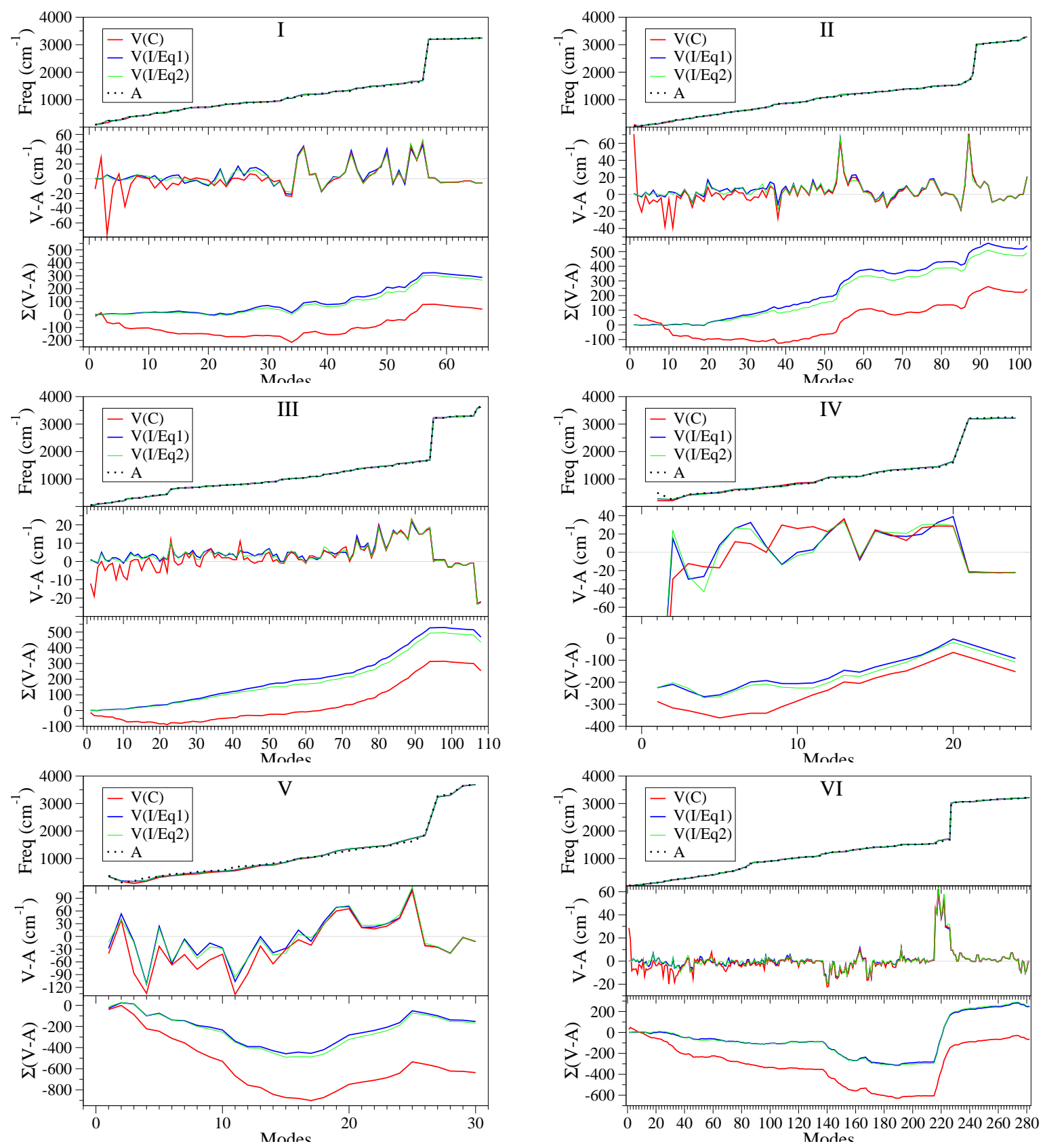

Figure S16: ES frequencies computed with the adiabatic (A), and vertical approach with Cartesian coordinates $(\mathrm{V}(\mathrm{C})$ ) and with internal ones using the $\mathbf{G}$ matrix evaluated at the initial state $(\mathrm{V}(\mathrm{I} / \mathrm{Eq} 1))$ or final state $(\mathrm{V}(\mathrm{I} / \mathrm{Eq} 2))$ minima. The differences between the adiabatic and vertical frequencies are shown in the middle panel, and the cumulative sum of the difference is included in the bottom panel. 


\section{S7 Conversion of shifts in internal coordinates shifts in Cartesian coordinates}

The transformation is done iteratively starting from the first order expression that relates $\Delta \mathbf{x}$ and $\Delta \mathbf{s}$, which can be derived from $\Delta \mathbf{s}=\mathbf{B} \Delta \mathbf{x}=\mathbf{W} \Delta \mathbf{q}$, where $\mathbf{W}=\mathbf{B M}^{-1 / 2}$. Taking the expression that depends on the mass-weighted Cartesian coordinates, $\mathbf{q}$, multiplying on the left by $\mathbf{W} \mathbf{W}^{+}$(where ${ }^{+}$denotes the pseudoinverse as defined in the paper) and taking into account that the pseudo-inverse fulfils $\mathbf{W} \mathbf{W}^{+} \mathbf{W}=\mathbf{W}$, the inverse relation reads, $\Delta \mathbf{q}=\mathbf{W}^{+} \Delta \mathbf{s}$, which once converted back to Cartesian coordinates, $\mathbf{x}$, can be written as, ${ }^{14,15}$

$$
\mathbf{x}_{1}^{(i)}=\mathbf{x}_{0}^{(i)}+\mathbf{M}^{-1} \mathbf{B}^{t} \mathbf{G}^{+} \Delta \mathbf{s}_{t}^{(i)}
$$

where $\mathbf{G}^{+}$is the pseudo-inverse of the $\mathbf{G}$ matrix $\left(\mathbf{G}=\mathbf{B} \mathbf{M}^{-1} \mathbf{B}^{t}\right), \mathbf{x}_{0}^{(i)}$ and $\mathbf{x}_{1}^{(i)}$ are the Cartesian coordinates before and after the displacement in the $i$-th iteration, and $\Delta \mathbf{s}_{t}^{(i)}$ is the targeted shift at the same iteration. In the first iteration, $\Delta \mathbf{s}_{t}^{(1)}$ is equal to the shift given by Eq. (16) in the paper, while in the subsequent steps it is equal to the difference between the targeted and actual shift between the geometries in Cartesian coordinates for the last iteration, $\Delta \mathbf{s}_{t}^{(i)}=\Delta \mathbf{s}_{t}^{(i-1)}-\Delta \mathbf{s}_{g}^{(i-1)}$, where $\Delta \mathbf{s}_{g}^{(i-1)}=\mathbf{s}\left(\mathbf{x}_{1}^{(i-1)}\right)-\mathbf{s}\left(\mathbf{x}_{0}^{(i-1)}\right)$. At each iteration, $\mathbf{x}_{0}$ is replaced by $\mathbf{x}_{1}$ and the $\mathbf{B}$ and $\mathbf{G}^{+}$matrices are updated. It should be noted that this transformation does not always reach convergence. ${ }^{14-16}$ A more straightforward approach to apply the transformation consists in picking a subset of internal coordinates that forms a Zmatrix among the redundant set, and then use the Z-matrix prescriptions to get the geometry. Regardless the strategy used, our results indicate that it is not possible to construct a geometry where all values at the final $\Delta \mathbf{s}_{\mathbf{g}}$ exactly match the targeted ones. Comparing the iterative method and the one based on a Z-matrix subset, it is observed that the iterative strategy tends to distribute more homogeneously the deviations among all coordinates, while in the Z-matrix approach, the shifts related to the Z-matrix subset are exactly defined, and the error on the rest of coordinates increases. This is the case for the molecules studied in this work. However, both procedures provide, in general, small deviations from the targeted displacements, $\Delta \mathbf{s}_{t}$, and actual values obtained in the Cartesian geometries, $\Delta \mathbf{s}_{g}$, as we report in the Tables S4 and S5. Namely, the maximum root mean square deviations, which are found for the valence angles in molecule $\mathbf{V}$, are $0.04^{\circ}$ for the iterative method and $0.14^{\circ}$ with the Z-matrix subset protocol. 
Table S4: Differences between the computed shift in redundant internal coordinates and the actual values once it is translated to Cartesian coordinates using a Z-matrix subset. The root mean square deviations (RMSD) and maximum absolute deviations (MAD) are reported for each type of coordinate.

\begin{tabular}{lcclccccc}
\hline \hline \multirow{2}{*}{ Molecule } & \multicolumn{2}{c}{ Bonds $(\AA)$} & & \multicolumn{2}{c}{ Angles(deg) } & & \multicolumn{2}{c}{ Dihed.(deg) } \\
\cline { 2 - 3 } \cline { 8 - 9 } & RMSD & MAD & & RMSD & MAD & & RMSD & MAD \\
\hline I & 0.000 & 0.000 & & 0.008 & 0.018 & & 0.000 & 0.000 \\
II & 0.002 & 0.010 & & 0.046 & 0.154 & & 0.074 & 0.247 \\
III & 0.000 & 0.000 & & 0.000 & 0.000 & & 0.000 & 0.001 \\
IV & 0.000 & 0.000 & & 0.000 & 0.000 & & 0.000 & 0.000 \\
V & 0.000 & 0.001 & & 0.139 & 0.294 & & 0.001 & 0.001 \\
VI & 0.000 & 0.001 & & 0.005 & 0.029 & & 0.004 & 0.018 \\
\hline \hline
\end{tabular}

Table S5: Differences between the computed shift in redundant internal coordinates and the actual values once it is translated to Cartesian coordinates using the iterative method. The root mean square deviations (RMSD) and maximum absolute deviations (MAD) are reported for each type of coordinate.

\begin{tabular}{lcccccccc}
\hline \hline & \multicolumn{2}{c}{ Bonds $(\AA)$} & & \multicolumn{2}{c}{ Angles(deg) } & & \multicolumn{2}{c}{ Dihed.(deg) } \\
\cline { 2 - 3 } Molecule & RMSD & MAD & & RMSD & MAD & & RMSD & MAD \\
\hline I & 0.000 & 0.000 & & 0.003 & 0.005 & & 0.000 & 0.000 \\
II & 0.000 & 0.001 & & 0.029 & 0.167 & & 0.020 & 0.090 \\
III & 0.000 & 0.000 & & 0.000 & 0.000 & & 0.000 & 0.001 \\
IV & 0.000 & 0.000 & & 0.000 & 0.000 & & 0.000 & 0.000 \\
V & 0.000 & 0.000 & & 0.039 & 0.081 & & 0.000 & 0.001 \\
VI & 0.000 & 0.000 & & 0.003 & 0.014 & & 0.002 & 0.007 \\
\hline \hline
\end{tabular}




\section{S8 Further comparisons of spectra simulations with vertical models}

\section{S8.1 Impact of the choice of the a real values to spurious imaginary frequencies (molecule II)}

The use of the vertical model with Cartesian coordinates can lead to spurious imaginary frequencies, an issue that can be solved by assigning an arbitrary real frequency. In order to get a more clear view on the impact of this choice, in Figure S17, we show the spectra simulated at 0 and $300 \mathrm{~K}$ by setting the two imaginary frequencies that arise with the $\mathrm{VH}(\mathrm{C})$ model for molecule II to different arbitrary values.

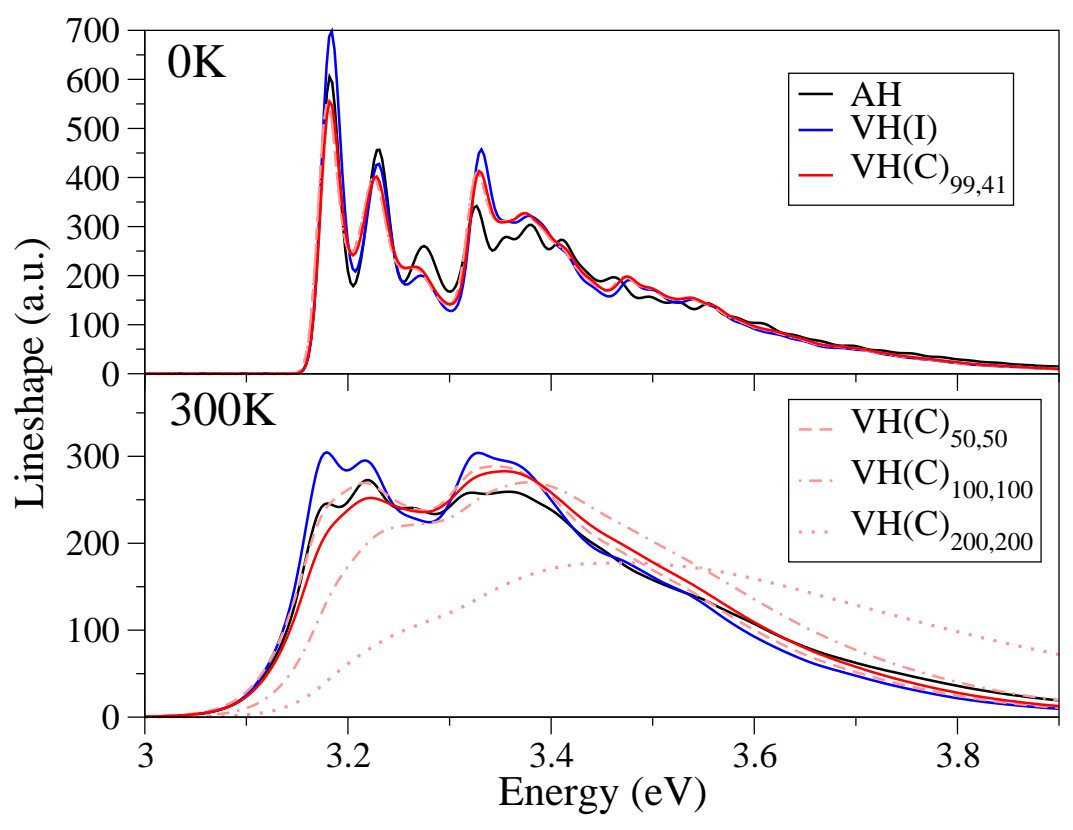

Figure S17: Lineshape spectra at 0 and $300 \mathrm{~K}$ simulated with the $\mathrm{AH}, \mathrm{VH}(\mathrm{I})$ and $\mathrm{VH}(\mathrm{C})$ models. For the latter, different choice are taken to assign real frequencies to the imaginary modes, $\mathrm{VH}(\mathrm{C})_{\nu_{1}, \nu_{2}}$. The spectra are broadened by a Gaussian function with $\mathrm{HWHM}=0.01 \mathrm{eV}$.

As observed in this figure, all choices lead to the same results at $0 \mathrm{~K}$. However, at $300 \mathrm{~K}$, the different choices (from 50 to $200 \mathrm{~cm}^{-1}$ ) lead to significantly different results. Interestingly, the adoption of the absolute values of the original imaginary frequencies (91 and $41 \mathrm{~cm}^{-1}$ ) lead to a result quite similar to the $\mathrm{AH}$ model, although other choices, such as setting both frequencies to $50 \mathrm{~cm}^{-1}$. On the other hand, as the frequencies increase, the discrepancies with the AH model (and $\mathrm{VH}(\mathrm{I})$ ) increase. 


\section{S8.2 Comparison with experimental data}

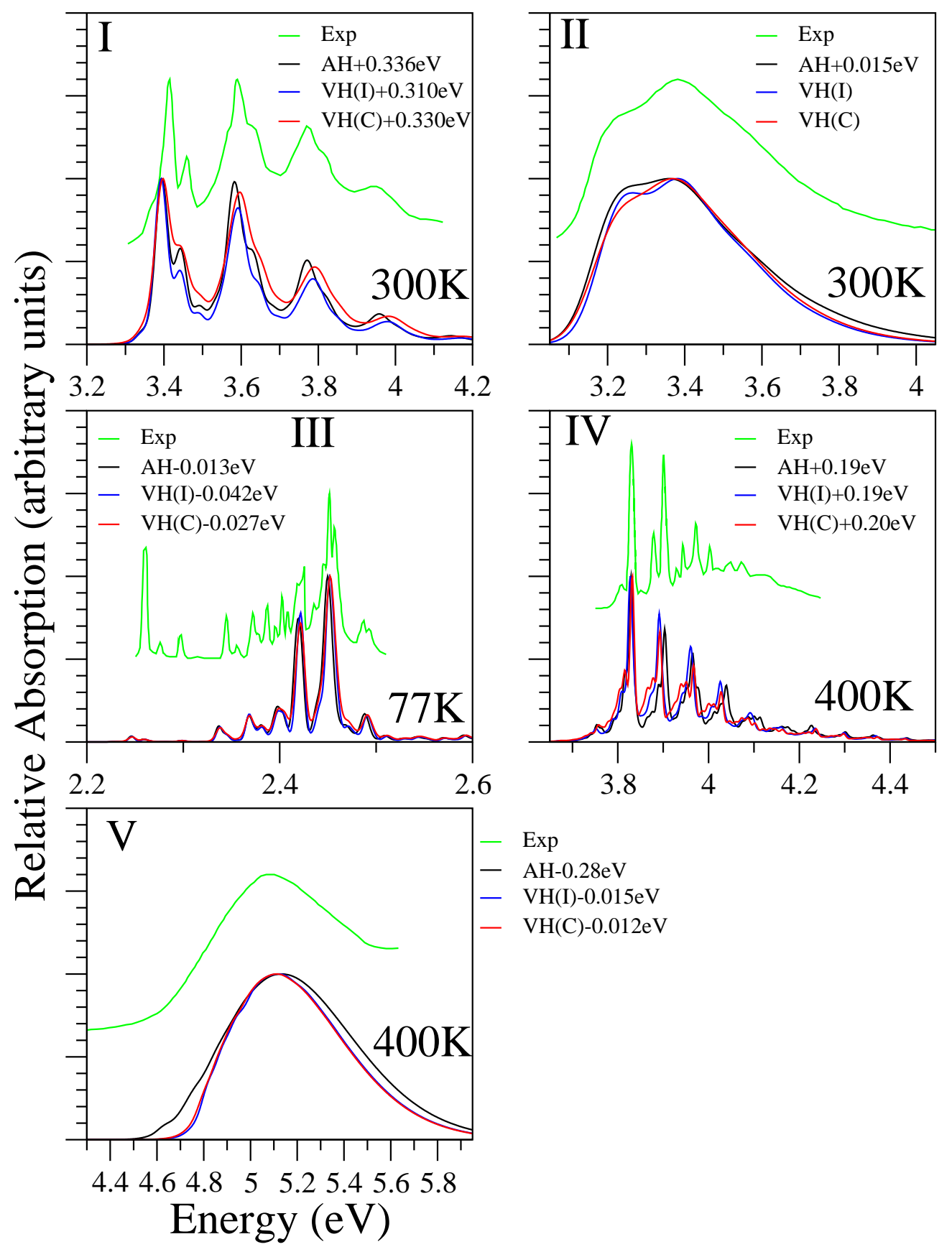

Figure S18: Absorption spectra of molecules I-V simulated with $\mathrm{AH}, \mathrm{VH}(\mathrm{I})$ and $\mathrm{VH}(\mathrm{C})$ models along with the FC approximation, except for III, for which the FCHT approach is used (see text). The spectra are broadened with a Gaussian convolution, with the FWHM taken from ref. 6. The experimental spectra from refs. 17 (I), 18 (II), 19 (III), 20 (IV) and 21 (V). The experimental spectra are shifted along the y-axis to facilitate the comparison with the simulations. 
The comparison with the experimental spectra is generally acceptable for all models, as already reported in for $\mathrm{AH}$ and $\mathrm{VH}(\mathrm{C})$ ones. ${ }^{6}$ However, there some aspects that need some further comments.

For instance, in the case of molecule III (porphyrin), the intensity of the overtones is enhanced by HT effects, while it is observed that the $0-0$ band is significantly underestimated with respect to the experimental one. This

We focus now on molecule IV (pyrazine). In this case, the final state PES was evaluated at a saddle point by constraining the symmetry to the same point group as the initial state minimum $\left(\mathrm{C}_{s}\right)$. Such a situation is an indication of a double-well potential, which is intrinsically anharmonic. In the paper, we kept the same general protocol for all cases where imaginary frequencies arise, in which the imaginary frequencies are transformed to real by taking their absolute values. For molecule $\mathbf{V}$, however, given the large differences between imaginary frequencies with vertical and adiabatic models, such a procedure might be unreliable when making the comparison with the experiment. This problem was already solved in a previous work by one of us ${ }^{6}$ by taking an effective harmonic frequency for this mode, estimated from a fitting of the 1D profile of the mode. The proposed value for the frequency is $1030 \mathrm{~cm}^{-1},{ }^{6}$ and it is the one adopted in this comparison with the experiment. 


\section{S9 Dependence on $\mathrm{G}_{\mathrm{eq}}$}
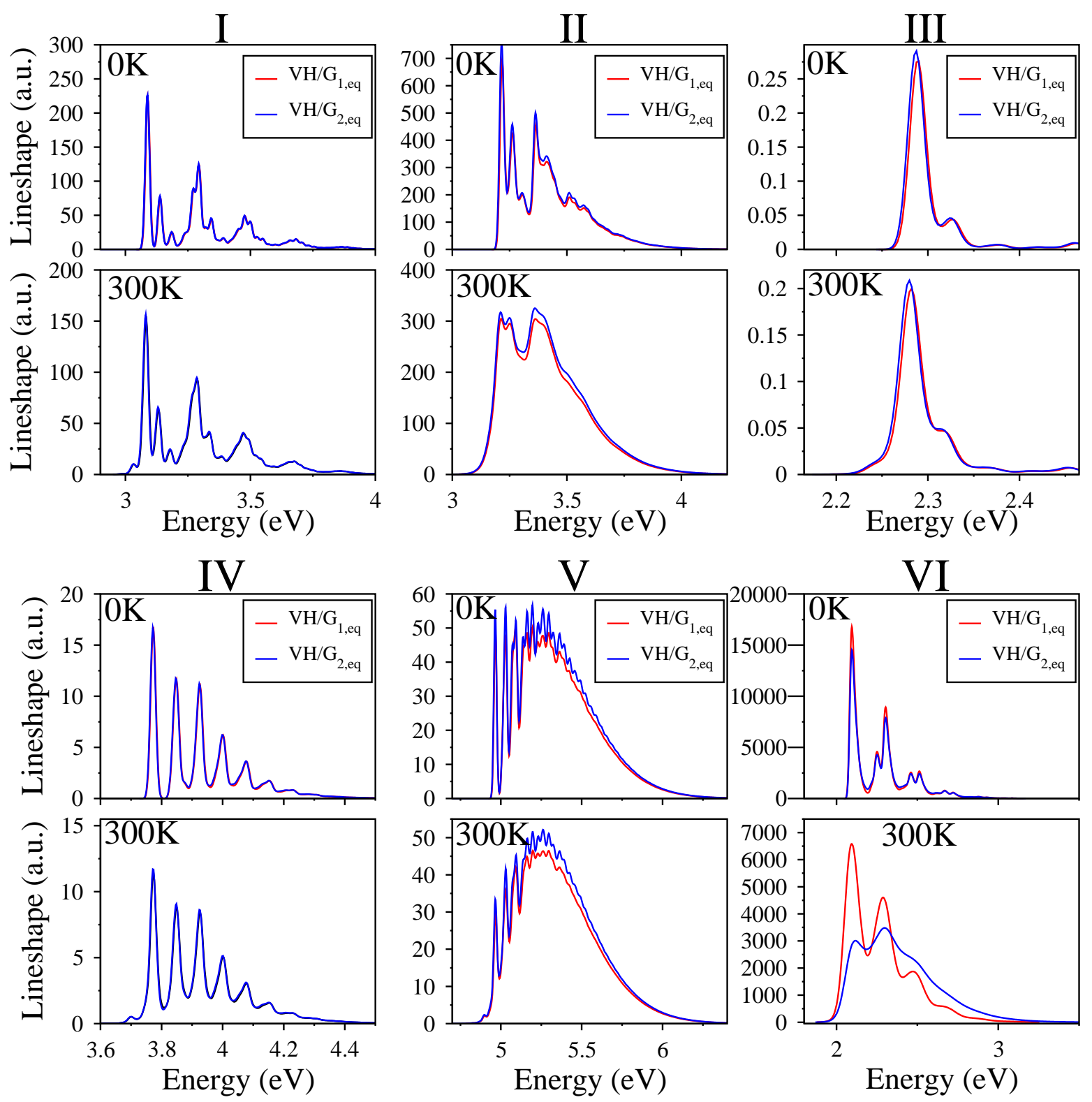

Figure S19: Lineshape spectra at 0 and $300 \mathrm{~K}$ simulated with the VH model in internal coordinates, adopting, for the normal mode analysis on the final state, the $\mathbf{G}$ matrix evaluated at the equilibrium of either the initial $\left(\mathbf{G}_{\mathbf{1}, \mathbf{e q}}\right)$ or final $\left(\mathbf{G}_{\mathbf{2} \text {,eq }}\right)$ states. The spectra are broadened by a Gaussian function with $\mathrm{HWHM}=0.01 \mathrm{eV}$. 


\section{References}

[1] Eckart, C. Some Studies Concerning Rotating Axes and Polyatomic Molecules. Phys. Rev. 1935, 47, 552-558.

[2] Sayvetz, A. The Kinetic Energy of Polyatomic Molecules. J. Chem. Phys. 1939, 7, 383-389.

[3] Wilson, E. B.; Decius, J. C.; Cross, P. C. Molecular Vibrations. The Theory of Infrared and Raman Vibrational Spectra; Dover, 1955.

[4] Kolossváry, I.; McMartin, C. On the degeneracy of the Hessian matrix. J. Math. Chem. 1992, 9, 359-367.

[5] Grochowski, P. Rotational symmetry of the molecular potential energy in the Cartesian coordinates. Theor. Chem. Acc. 2008, 121, 257-266.

[6] Avila Ferrer, F. J.; Santoro, F. Comparison of vertical and adiabatic harmonic approaches for the calculation of the vibrational structure of electronic spectra. Phys. Chem. Chem. Phys. 2012, 14, 13549-13563.

[7] Califano, S. Vibrational States; J. Wiley and Sons, Ltd.: London, 1976; Chapter 4.

[8] Hazra, A.; Chang, H. H.; Nooijen, M. First principles simulation of the UV absorption spectrum of ethylene using the vertical Franck-Condon approach. J. Chem. Phys. 2004, 121, 2125-2136.

[9] Götze, J. P.; Karasulu, B.; Thiel, W. Computing UV/vis spectra from the adiabatic and vertical Franck-Condon schemes with the use of Cartesian and internal coordinates. J. Chem. Phys. 2013, 139, 234108.

[10] Baiardi, A.; Bloino, J.; Barone, V. General formulation of vibronic spectroscopy in internal coordinates. J. Chem. Phys. 2016, 144, 084114.

[11] Koch, W.; Holthausen, M. C. A Chemist's Guide to Density Functional Theory; Wiley-VCH Verlag GmbH, 2001.

[12] Wales, D. J. Potential energy surfaces and coordinate dependence. J. Chem. Phys. 2000, 113, 3926-3927.

[13] Brandhorst, K.; Grunenberg, J. Efficient computation of compliance matrices in redundant internal coordinates from Cartesian Hessians for nonstationary points. J. Chem. Phys. 2010, 132, 184101.

[14] Pulay, P.; Fogarasi, G. Geometry optimization in redundant internal coordinates. J. Chem. Phys. 1992, 96, 2856-2860. 
[15] Peng, C.; Ayala, P. Y.; Schlegel, H. B.; Frisch, M. J. Using redundant internal coordinates to optimize equilibrium geometries and transition states. $J$. Comput. Chem. 1996, 17, 49-56.

[16] Bakken, V.; Helgaker, T. The efficient optimization of molecular geometries using redundant internal coordinates. J. Chem. Phys. 2002, 117, 9160-9174.

[17] Ferguson, J.; Reeves, L. W.; Schneider, W. G. Vapor absorption spectra and oscillator strengths of naphthalene, anthracene, and pyrene. Can. J. Chem. 1957, 35, 1117-1136.

[18] Muhlpfordt, A.; Schanz, R.; Ernsting, N.; Farztdinov, V.; Grimme, S. Coumarin 153 in the gas phase: optical spectra and quantum chemical calculations. Phys. Chem. Chem. Phys. 1999, 1, 3209-3218.

[19] Gradyushko, A. T.; Solovev, K. N.; Starukhin, A. S. Electronic-vibraional interaction and mirror symmetry of the quasi-line absorption and fluorescence specta of porphyrins. Part 1: porphin. Opt. Spektrosk. 1976, 40, 469.

[20] Suzuka, I.; Udagawa, Y.; Ito, M. Raman spectra of pyrazine resonant to the $\mathrm{S} 2\left(\pi, \pi^{*}\right)$ state and the geometry in excited state. Chem. Phys. Lett. 1979, $64,333-336$.

[21] Clark, L. B.; Peschel, G. G.; Jr., I. T. Vapor Spectra and Heats of Vaporization of Some Purine and Pyrimidine Bases1. J. Phys. Chem. 1965, 69, 3615-3618. 